\title{
Normalizers in groups and in their profinite completions
}

\author{
Luis Ribes and Pavel A. Zalesskil
}

\begin{abstract}
Let $R$ be a finitely generated virtually free group (a finite extension of a free group) and let $H$ be a finitely generated subgroup of $R$. Denote by $\hat{R}$ the profinite completion of $R$ and let $\bar{H}$ be the closure of $H$ in $\hat{R}$. It is proved that the normalizer $N_{\hat{R}}(\bar{H})$ of $\bar{H}$ in $\hat{R}$ is the closure in $\hat{R}$ of $N_{R}(H)$. The proof is based on the fact that $R$ is the fundamental group of a graph of finite groups over a finite graph and on the study of the minimal $H$-invariant subtrees of the universal covering graph of that graph of groups. As a consequence we prove results of the following type: let $R$ be a group that is an extension of a free group by finite solvable group, and let $x, y \in R$; then $x$ and $y$ are conjugate in $R$ if their images are conjugate in every finite quotient of $R$.
\end{abstract}

Let $R$ be a residually finite abstract group. Then $R$ is embedded naturally in its profinite completion

$$
\hat{R}=\underbrace{\lim }_{U \in \mathcal{U}} R / U
$$

where $\mathcal{U}$ denotes the collection of all normal subgroups $U$ of finite index in $R$. Given a subset $X$ of $R$, denote its topological closure in $\hat{R}$ by $\bar{X}$. This paper is concerned with the following problem: if $H$ is a finitely generated subgroup of $R$, what is the relationship between the normalizer $N_{R}(H)$ of $H$ in $R$ and the normalizer $N_{\hat{R}}(\bar{H})$ of $\bar{H}$ in $\hat{R}$ ? Originally this question arose in [10] while studying conjugacy separability in groups that arise as iterations of amalgamated free products of certain groups. In [10] the question is answered when $R$ is a finite extension of a polycyclic group; and then the answer is the desirable one: $N_{\hat{R}}(\bar{H})$ is the closure in $\hat{R}$ of $N_{R}(H)$. Perhaps not completely surprising given the nature of polycyclic groups, the proof of that result is 'arithmetic' and eventually it relies on number theoretic results and methods.

In this paper we deal with the case when $R$ is a finitely generated abstract virtually free group, i.e., a finite extension of a free group $\Phi$. Such a group is residually finite. In fact our results are placed in a more general setting: we

Mathematics Subject Classification (2010): 20E18, $20 \mathrm{E} 08$.

Keywords: Residually finite groups, profinite groups, normalizer, conjugacy separability. 
consider a group $R$ that contains a free normal subgroup $\Phi$ such that $R / \Phi$ is a group in a given class $\mathcal{C}$ of finite groups which is an extension-closed variety of finite groups, i.e., $\mathcal{C}$ is a nonempty class of finite groups closed under subgroups, homomorphic images and extensions. For example, $\mathcal{C}$ could be the class of all finite groups, the class of all finite solvable groups, or the class of all finite $p$ groups, where $p$ is a fixed prime number. Then instead of the profinite completion of $R$ we study the problem mentioned above for the pro- $\mathcal{C}$ completion

$$
R_{\hat{\mathcal{C}}}=\underbrace{}_{U \triangleleft R, R / U \in \mathcal{C}} R / U
$$

of $R$. It turns out that in this case $R$ is also canonically embedded in $R_{\hat{\mathcal{C}}}$, so that the question mentioned above has a natural analog in this new setting. The answer is again formally the same: $N_{R_{\hat{\mathcal{C}}}}(\bar{H})$ is the closure in $R_{\hat{\mathcal{C}}}$ of $N_{R}(H)$. However the methods are completely different. In this case one needs a 'combinatorial' approach based on the Bass-Serre theory [16] of groups acting on trees and its counterpart for profinite groups [4], [18]. By results of Serre (cf. Part II, Proposition 12 in [16]) and Karrass, Pietrowski and Solitar (cf. Theorem 1 in [7]), finitely generated virtually free groups are precisely the fundamental groups $\Pi_{1}^{\text {abs }}(\mathcal{G}, \Delta)$ of graphs of finite groups over a finite graph $\Delta$. In the case we consider here the vertex groups of this graph of groups are in the class $\mathcal{C}$. Associated with a graph of groups there is a tree $S^{\text {abs }}$, its universal (or standard) covering on which $\Pi_{1}^{\text {abs }}(\mathcal{G}, \Delta)$ operates. Similarly there is a pro- $\mathcal{C}$ fundamental group $\Pi_{1}(\mathcal{G}, \Delta)$ associated with this graph of finite groups, and a corresponding pro- $\mathcal{C}$ tree $S$ which is also a topological space. In the case we are interested in, $\Pi_{1}(\mathcal{G}, \Delta)$ is the pro- $\mathcal{C}$ completion of $\Pi_{1}^{\text {abs }}(\mathcal{G}, \Delta)$, and $S^{\text {abs }}$ is embedded as a dense subgraph of $S$.

Our method, which is perhaps the key original contribution of the present paper that makes the proofs work, is based on the study of the relationship between these two trees. First we show that for a closed (in the pro- $\mathcal{C}$ topology) finitely generated infinite subgroup $H$ of $\Pi_{1}^{\text {abs }}(\mathcal{G}, \Delta), S^{\text {abs }}$ contains a unique minimal $H$-invariant subtree whose closure in $S$ is the unique minimal $\bar{H}$-invariant pro- $\mathcal{C}$ subtree of $S$. This leads to the proof of our main result when $H$ is infinite. When $H$ is finite, the basic result needed, that has independent interest, is that $\overline{H_{1} \cap H_{2}}=\overline{H_{1}} \cap \overline{H_{2}}$ in $R_{\hat{\mathcal{C}}}$, for closed, finitely generated subgroups $H_{1}$ and $H_{2}$ of $R$. This is proved again using combinatorial methods: the key argument is based on the study of the tree canonically attached to an amalgamated product of abstract groups, and its counterpart in the category of pro- $\mathcal{C}$ groups.

We also apply our method to study the Tits straight line $L_{b}$ in a tree (cf. Serre [16], Part I, Prop. 24) of the form $S^{\text {abs }}$ corresponding to the graph of groups $(\mathcal{G}, \Delta)$ of residually finite groups $\mathcal{G}(m)(m \in \Delta)$ over a finite graph $\Delta ; L_{b}$ is the minimal $\langle b\rangle$-invariant subtree of $S^{\text {abs }}$ where $b \in \Pi_{1}^{\text {abs }}(\mathcal{G}, \Delta)$ acts freely on $S^{\text {abs }}$. We prove that then $\overline{L_{b}}$ is the unique minimal $\overline{\langle b\rangle}$-invariant subtree of the profinite tree $S$, where $S$ is the universal covering profinite tree associated with the graph of profinite groups $(\overline{\mathcal{G}}, \Delta)$ such that each $\overline{\mathcal{G}}(m)$ is a profinite completion of $\mathcal{G}(m)$. We accomplish this by first showing that the profinite fundamental group $\Pi_{1}(\overline{\mathcal{G}}, \Delta)$ can be expressed as an inverse limit of virtually free profinite groups. 
We finish the paper with an application of the above results to the following version of conjugacy separability. Let $R$ be a finitely generated abstract group that contains a normal free subgroup $\Phi$ such that $R / \Phi$ is in a fixed extension-closed variety of finite groups $\mathcal{C}$. Then we show that two elements $x$ and $y$ of $R$ are conjugate in $R$ if and only if their images in every quotient group $C$ of $R$ that is in $\mathcal{C}$ are conjugate; in other words, $x$ and $y$ are conjugate in $R$ if and only if they are conjugate in $R_{\hat{\mathcal{C}}}$. The result was known (proved by Baumslag and Taylor) when $R=\Phi$ is a free abstract group (cf. Proposition 4.8 in [8]). When $\mathcal{C}$ is the class of all finite groups, this is the well-known result that free-by-finite groups are conjugacy separable (Dyer [2]), and it was proved by Toinet [17] when $\mathcal{C}$ is the variety of finite groups consisting of all finite $p$-groups, for a fixed prime number $p$.

\section{Notation}

Generally, we follow the notation of [14], which can be consulted for the main concepts and results related to profinite groups used in this paper; alternatively, the reader may consult [15], for example. Throughout the paper we shall assume that $\mathcal{C}$ is a variety of finite groups closed under extensions, i.e., $\mathcal{C}$ is a nonempty collection of (isomorphism classes of) finite groups closed under the operations of taking subgroups, homomorphic images and extensions. For example, $\mathcal{C}$ can be the class of all finite groups, the class of all finite $p$-groups for a fixed prime number $p$, or the class of all finite solvable groups. A 'pro- $\mathcal{C}$ group' $G$ is an inverse limit of groups in $\mathcal{C}$; this is a compact, Hausdorff, and totally-disconnected topological group with the property $G / U \in \mathcal{C}$, whenever $U$ is an open normal subgroup of $G$.

Let $R$ be an abstract group. Recall that the (full) 'pro- $\mathcal{C}$ topology' of $R$ is the unique topology that makes $R$ into a topological group in such a way that the set $\mathcal{U}$ of all normal subgroups $U$ of $R$ with $R / U \in \mathcal{C}$ form a fundamental system of neighbourhoods of the identity element 1 . One says that $R$ is 'residually $\mathcal{C}$ ' if this topology is Hausdorff, i.e., if $\bigcap_{U \in \mathcal{U}} U=1$. The 'pro- $\mathcal{C}$ completion' $R_{\hat{\mathcal{C}}}$ of $R$ is the pro- $\mathcal{C}$ group

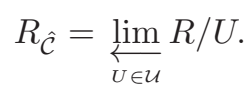

The natural homomorphism $R \longrightarrow R_{\hat{\mathcal{C}}}$ is continuous ( $R$ is endowed with its pro- $\mathcal{C}$ topology). If $R$ is residually $\mathcal{C}$, this homomorphism is an injection, and we identify $R$ with its image in $R_{\hat{\mathcal{C}}}$, so that $R \leq R_{\hat{\mathcal{C}}}$. In this case the topology on $R$ induced by the topology of $R_{\hat{\mathcal{C}}}$ is precisely its pro- $\mathcal{C}$ topology. If $X \subseteq R$, we denote the closure of $X$ in $R$ by $C l(X)$, and the closure of $X$ in $R_{\hat{\mathcal{C}}}$ by $\bar{X}$; we note that $C l(X)=R \cap \bar{X}$ and $\bar{X}=\overline{C l(X)}$ (cf. Section 3 of [11]).

If $A$ and $B$ are pro- $\mathcal{C}$ groups, we will denote by $A \amalg B$ their free pro- $\mathcal{C}$ product, i.e., their coproduct in the category of pro- $\mathcal{C}$ groups (cf. Section 9.1 of [14]).

We shall often be interested in abstract groups $R$ that are free-by-C ; that is, $R$ contains a normal free subgroup $\Phi$ such that $R / \Phi \in \mathcal{C}$, or equivalently, $\Phi$ is open in the pro- $\mathcal{C}$ topology of $R$. It turns out that such a group $R$ is residually $\mathcal{C}$ (see Lemma 0.4 below). When $\mathcal{C}$ is the class of all finite groups, we revert to the usual terminology 'free-by-finite' (or 'virtually free') rather than free-by- $\mathcal{C}$. 


\section{Preliminaries}

We begin by recalling the definitions of the fundamental group and universal covering graph of a graph of groups, using a language and a notation common to the abstract and profinite settings that is convenient for our purposes. With this in mind, we shall not attempt to give these definitions in the most general setting. Instead we shall consider only graphs of groups $(\mathcal{G}, \Delta)$ over finite connected graphs $\Delta$ (in the abstract case this does not make an important difference, but in the profinite case the finiteness of $\Delta$ makes the definitions much simpler). We also include here some consequence of well-known results. We refer to the papers [4] and [20] for basic definitions and results.

We only consider oriented graphs (cf. Chapter I of [1]; in the language of [16], we choose a specific 'orientation' of a graph). A graph $\Gamma$ is a set together with a distinguished subset of 'vertices' $V=V(\Gamma)$ together with two maps $d_{0}, d_{1}: \Gamma \longrightarrow V$, that restrict to the identity on $V$. This graph is called 'profinite' if $\Gamma$ is a profinite space (i.e., a compact, Hausdorff, and totally-disconnected topological space), $V$ is a closed subset of $\Gamma$, and the mappings $d_{i}$ are continuous. If $e \in \Gamma$, we say that $d_{0}(e)$ and $d_{1}(e)$ are the origin and terminal vertex of $e$, respectively. $E=E(\Gamma)=\Gamma-V(\Gamma)$ is called the set (space) of 'edges' of $\Gamma$. For basic concepts such as connectedness or when a graph is a tree see Chapter I of [1] or Part I of [16], for abstract graphs; and in the profinite case, [4] or [20]. If $v$ and $w$ are elements of a tree (respectively, a pro- $\mathcal{C}$ tree) $T$, we denote by $[v, w]$ the smallest subtree (respectively, pro- $\mathcal{C}$ subtree) of $T$ containing $v$ and $w$.

A group $H$ is said to act on a graph $\Gamma$ if it acts on it as a set and in addition $d_{i}(h m)=h d_{i}(m)$, for all $h \in H$ and $m \in \Gamma(i=0,1)$; if $\Gamma$ is a profinite graph and $H$ a profinite group, we assume that the action is continuous. The quotient $H \backslash \Gamma$ inherits a natural graph structure (respectively, profinite graph structure).

Let $\Delta$ be a connected finite graph. A 'graph of groups' $(\mathcal{G}, \Delta)$ over $\Delta$ consists of a group $\mathcal{G}(m)$ for each $m \in \Delta$, and monomorphisms $\partial_{i}: \mathcal{G}(e) \longrightarrow \mathcal{G}\left(d_{i}(e)\right)$ for each edge $e \in E(\Delta)$. If each $\mathcal{G}(m)$ is a pro- $\mathcal{C}$ group and the monomorphisms $\partial_{i}$ are continuous, we say that $(\mathcal{G}, \Delta)$ is a 'graph of pro- $\mathcal{C}$ groups'. The 'abstract fundamental group'

$$
\Pi^{\mathrm{abs}}=\Pi_{1}^{\mathrm{abs}}(\mathcal{G}, \Delta)
$$

of the graph of groups $(\mathcal{G}, \Delta)$ is defined by means of a universal property. Namely, $\Pi^{\text {abs }}$ is an abstract group together with the following data and conditions:

(i) a maximal subtree $T$ of $\Delta$;

(ii) a collection of homomorphisms

$$
\nu_{m}: \mathcal{G}(m) \longrightarrow \Pi^{\mathrm{abs}}(m \in \Delta),
$$

and a map $E(\Delta) \longrightarrow \Pi^{\text {abs }}$, written $e \mapsto t_{e}(e \in E(\Delta))$, such that $t_{e}=1$, if $e \in E(T)$, and

$$
\left(\nu_{d_{0}(e)} \partial_{0}\right)(x)=t_{e}\left(\nu_{d_{1}(e)} \partial_{1}\right)(x) t_{e}^{-1}, \quad \forall x \in \mathcal{G}(e), e \in E(\Delta) ;
$$


(iii) the following universal property is satisfied: whenever one has the data

- $H$ is an abstract group,

- $\beta_{m}: \mathcal{G}(m) \longrightarrow \Pi^{\mathrm{abs}}(m \in \Delta)$ a collection of homomorphisms,

- a map $e \mapsto s_{e}(e \in E(\Delta))$ with $s_{e}=1$, if $e \in E(T)$,

- and

$$
\left(\beta_{d_{0}(e)} \partial_{0}\right)(x)=s_{e}\left(\beta_{d_{1}(e)} \partial_{1}\right)(x) s_{e}^{-1}, \quad \forall x \in \mathcal{G}(e), e \in E(\Delta),
$$

then there exists a unique homomorphism $\delta: \Pi^{\text {abs }} \longrightarrow H$ such that $\delta\left(t_{e}\right)=s_{e}$ $(e \in E(\Delta))$, and for each $m \in \Delta$ the diagram

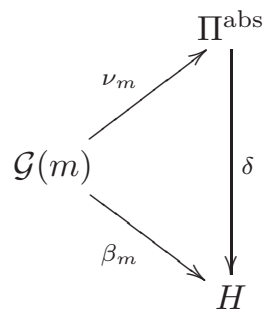

commutes.

In Chapter I, Definition 7.3 and Corollary 7.5 of [1], and in Part I, Sections 5.1 and 5.2 of [16], the fundamental group $\Pi^{\text {abs }}$ is defined explicitly in terms of generators and relations; there it is also proved that the definition given above is independent of the choice of the maximal subtree $T$, and furthermore it is proved that the homomorphisms $\nu_{m}: \mathcal{G}(m) \longrightarrow \Pi^{\text {abs }}$ are injective for every $m \in \Delta$. We use the notation $\Pi^{\text {abs }}(m)=\operatorname{Im}\left(\nu_{m}\right)$; so $\Pi^{\text {abs }}(m) \cong \mathcal{G}(m)$, for $m \in \Delta$.

The definition of the 'pro- $\mathcal{C}$ fundamental group'

$$
\Pi=\Pi_{1}(\mathcal{G}, \Delta)
$$

of a graph $(\mathcal{G}, \Delta)$ of pro- $\mathcal{C}$ groups over a finite graph $\Delta$ is formally as before: one simply assumes that all the conditions take place in the category of pro- $\mathcal{C}$ groups, i.e., all groups involved are pro- $\mathcal{C}$ and all homomorphisms are assumed to be continuous. For the construction of $\Pi$, see [20]. However, the canonical homomorphisms $\nu_{m}: \mathcal{G}(m) \longrightarrow \Pi(m \in \Delta)$ are not embeddings in general (cf. Examples 9.2.9 and 9.2.10 in [14]). We use the notation $\Pi(m)=\operatorname{Im}\left(\nu_{m}\right)$ for $m \in \Delta$.

Associated with the graph of groups $(\mathcal{G}, \Delta)$ there is a corresponding standard graph (or universal covering graph) $S^{\text {abs }}=\bigcup \Pi^{\text {abs }} / \Pi^{\text {abs }}(m)$, and the vertices of $S^{\text {abs }}$ are those cosets of the form $g \Pi^{\text {abs }}(v)$, with $v \in V(\Delta)$ and $g \in \Pi^{\text {abs }}$; finally, the incidence maps of $S^{\text {abs }}$ are given by the formulas:

$$
d_{0}\left(g \Pi^{\mathrm{abs}}(e)\right)=g \Pi^{\mathrm{abs}}\left(d_{0}(e)\right) ; \quad d_{1}\left(g \Pi^{\mathrm{abs}}(e)\right)=g t_{e} \Pi^{\mathrm{abs}}\left(d_{1}(e)\right) \quad(e \in E(\Delta)) .
$$


In fact $S^{\text {abs }}$ is a tree (cf. Chapter I, Theorem 7.6 in [1], or part I, Section 5.3 in [16]). There is a natural left action of $\Pi^{\text {abs }}$ on $S^{\text {abs }}$, and clearly $\Pi^{\text {abs }} \backslash S^{\text {abs }}=\Delta$. Analogously, there is a profinite standard graph $S=\bigcup \Pi / \Pi(m)$ associated with a graph of pro- $\mathcal{C}$ groups $(\mathcal{G}, \Delta)$, with space of vertices and with incidence maps defined as above. In fact $S$ is a pro- $\mathcal{C}$ tree (cf. [20]). $\Pi$ acts continuously on $S$ and $\Pi \backslash S=\Delta$.

Next we describe explicitly a useful connection between the standard tree $S^{\text {abs }}=S^{\text {abs }}(\mathcal{G}, \Delta)$ of a graph of abstract groups $(\mathcal{G}, \Delta)$ over a finite graph $\Delta$, and the standard pro- $\mathcal{C}$ tree $S=S(\overline{\mathcal{G}}, \Delta)$ of a graph of pro- $\mathcal{C}$ groups $(\overline{\mathcal{G}}, \Delta)$ that we described presently, after we make some basic assumptions. We shall assume that the fundamental group $\Pi^{\text {abs }}=\Pi_{1}^{\text {abs }}(\mathcal{G}, \Delta)$ is residually $\mathcal{C}$; we denote by $\Pi$ the pro- $\mathcal{C}$ completion of $\Pi^{\text {abs }}$. For each $m \in \Delta$, the pro- $\mathcal{C}$ topology of $\Pi^{\text {abs }}$ induces on $\Pi^{\text {abs }}(m)$ a certain pro- $\mathcal{C}$ topology (which is not necessarily its full pro- $\mathcal{C}$ topology) and so on $\mathcal{G}(m)$. Define $\overline{\mathcal{G}}(m)$ to be the completion of $\mathcal{G}(m)$ with respect to this topology. Then the monomorphisms $\partial_{i}: \mathcal{G}(e) \longrightarrow \mathcal{G}\left(d_{i}(e)\right)$ induce continuous monomorphisms which we again denote by $\partial_{i}: \overline{\mathcal{G}}(e) \longrightarrow \overline{\mathcal{G}}\left(d_{i}(e)\right)(i=0,1)$. We have then a graph $(\overline{\mathcal{G}}, \Delta)$ of pro- $\mathcal{C}$ groups over $\Delta$. The canonical injection $\mathcal{G}(m) \longrightarrow \Pi^{\text {abs }}$ induces an injection $\overline{\mathcal{G}}(m) \longrightarrow \Pi(m \in \Delta)$; furthermore, if we denote by $\Pi(m)$ the image of $\overline{\mathcal{G}}(m)$ on $\Pi$ under this injection, then $\Pi(m)=\overline{\Pi^{\text {abs }}(m)}$, the closure of $\Pi^{\mathrm{abs}}(m)$ in $\Pi$.

Clearly

$$
\partial_{0}(g)=t_{e} \partial_{1}(g) t_{e}^{-1} \quad(g \in \overline{\mathcal{G}}(e), e \in E(\Delta))
$$

in $\Pi$ (there a certain abuse of notation here, as we are identifying $\overline{\mathcal{G}}(v)$ with its image in $\Pi$, and similarly we are using the same notation for the original elements $t_{e}$ $(e \in E(\Delta))$ and their images in $\Pi$, which is justified since with our assumptions $\left.\Pi^{\text {abs }} \leq \Pi\right)$. Furthermore, one checks immediately the following result.

Proposition 0.1. The pro- $\mathcal{C}$ completion $\Pi$ of $\Pi^{\text {abs }}$ is the fundamental pro-C group $\Pi_{1}(\overline{\mathcal{G}}, \Delta)$ of the graph of pro-C groups $(\overline{\mathcal{G}}, \Delta)$. The canonical homomorphisms $\overline{\mathcal{G}}(m) \longrightarrow \Pi=\Pi_{1}(\overline{\mathcal{G}}, \Delta)$ are injective $(m \in \Delta)$.

We make a further assumption, namely that for each $m \in \Delta, \Pi^{\mathrm{abs}}(m)$ is closed in the pro- $\mathcal{C}$ topology of $\Pi^{\text {abs }}$ (or, equivalently, $\Pi(m) \cap \Pi^{\text {abs }}=\Pi^{\text {abs }}(m)$ ). Consider the natural morphism of graphs

$$
\varphi: S^{\mathrm{abs}} \longrightarrow S
$$

which on vertices and edges is

$$
g \Pi^{\mathrm{abs}}(v) \mapsto g \Pi(v), \quad g \Pi^{\mathrm{abs}}(e) \mapsto g \Pi(e) \quad\left(g \in \Pi^{\mathrm{abs}}, v \in V(\Delta), e \in E(\Delta)\right) .
$$

Under these assumptions $\varphi$ is an injection of graphs; we think of $S^{\text {abs }}$ as a subgraph of $S$. Moreover it is clear that $S^{\text {abs }}$ is dense in $S$. We collect all of this in the following proposition. 
Proposition 0.2. Let $(\mathcal{G}, \Delta)$ be a graph of abstract groups over a finite connected graph $\Delta$. Assume that its abstract fundamental group $\Pi^{\text {abs }}=\Pi_{1}^{\text {abs }}(\mathcal{G}, \Delta)$ is residually $\mathcal{C}$ and that each $\Pi^{\mathrm{abs}}(m)$ is closed in the pro- $\mathcal{C}$ topology of $\Pi_{1}^{\mathrm{abs}}(\mathcal{G}, \Delta)$. Consider the graph $(\overline{\mathcal{G}}, \Delta)$ of pro- $\mathcal{C}$ groups over $\Delta$ such that each $\overline{\mathcal{G}}(m)$ is the completion of $\mathcal{G}(m)$ with respect to the topology induced by the pro- $\mathcal{C}$ topology of $\Pi^{\text {abs }}$. Then the standard (or universal covering) tree $S^{\mathrm{abs}}=S^{\mathrm{abs}}(\mathcal{G}, \Delta)$ of the graph of groups $(\mathcal{G}, \Delta)$ is canonically embedded in the standard pro-C tree $S=S(\overline{\mathcal{G}}, \Delta)$ of the graph of pro-C groups $(\overline{\mathcal{G}}, \Delta)$, and $S^{\text {abs }}$ is dense in $S$.

Remark 0.3. The assumptions that we have made in Propositions 0.1 and 0.2 for the graph of groups $(\mathcal{G}, \Delta)$ and for the abstract fundamental group $\Pi^{\text {abs }}=$ $\Pi_{1}(\mathcal{G}, \Delta)$ are automatically satisfied if $\mathcal{C}$ is the variety of all finite groups and if the groups $\mathcal{G}(m)$ are finite for all $m \in \Delta$; indeed, in this case $\Pi^{\text {abs }}$ is residually finite (cf. Part II, Proposition 11 in [16]), and obviously the groups $\Pi^{\text {abs }}(m) \cong \mathcal{G}(m)$ are closed in the profinite topology of $\Pi^{\text {abs }}$ since they are finite. In this situation $\Pi^{\text {abs }}$ is in fact a finitely generated free-by-finite group. See Lemma 1.5 for a case valid for a more general variety of finite groups $\mathcal{C}$.

In the following lemma we consider a particular type of residually $\mathcal{C}$ groups that will be of interest to us later.

Lemma 0.4. Let $R$ be a finitely generated abstract free-by-C group: say $\Phi \triangleleft R, \Phi$ is a free group and $R / \Phi \in \mathcal{C}$. Then the pro-C topology of $R$ is Hausdorff, i.e., $R$ is residually $\mathcal{C}$.

Proof. Let $1 \neq x \in R$. We need to prove the existence of a normal subgroup $U$ of $R$ such that $R / U \in \mathcal{C}$ and $x \notin U$. If $x \notin \Phi$, choose $U=\Phi$. Assume $x \in \Phi$. The pro- $\mathcal{C}$ topology of $\Phi$ coincides with the topology induced by the pro- $\mathcal{C}$ topology of $R$ (cf. Lemma 3.1.4 (a) in [14]). On the other hand, the pro-C topology of a free abstract group is Hausdorff (cf. Proposition 3.3.15 in [14]). Hence there exists a normal subgroup $U$ of $R$ with $R / U \in \mathcal{C}, U \leq \Phi$ and $x \notin U$.

\section{Minimal subtrees}

In this section we study cases when the tree $S^{\text {abs }}$ has a unique minimal $H$-invariant subtree $D^{\text {abs }}$, and correspondingly when the pro-C tree $S$ has a unique $\bar{H}$-invariant pro- $\mathcal{C}$ subtree $D$. We are interested in the relationship between $D^{\text {abs }}$ and $D$. This will be a basic tool in our study of normalizers in the next section.

For ease of reference we first state some results obtained elsewhere.

Lemma 1.1 (Lemma 3.2 in [13]). Let $H$ be an abstract group that acts freely on an abstract tree $T$. Endow $H$ with its pro-C topology. Let $K$ be a closed subgroup of $H$ and let $\Delta$ be a finite subgraph of $K \backslash T$. Then there exists an open subgroup $V$ of $H$ containing $K$ such that the natural map of graphs

$$
\tau_{V}: K \backslash T \longrightarrow V \backslash T
$$

is injective on $\Delta$. 
Lemma 1.2. Let $H$ be a pro-C group that acts continuously on a pro-C tree $T$. Then

(a) $T$ has always a minimal $H$-invariant pro-C subtree $D$; moreover it is unique if $|D|>1$. In particular $D$ is unique if $H$ is infinite and the stabilizer of each vertex is finite.

(b) Assume that $H$ is infinite and the stabilizer of each vertex is finite. Let $D$ be the unique minimal $H$-invariant pro-C subtree of $T$, and let $N$ be an infinite normal subgroup of $H$. Then $D$ is also the unique minimal $N$-invariant pro-C subtree of $T$.

Proof. Part (a) appears in Lemma 1.5 of [19]. To prove (b), let $B$ be the unique minimal $N$-invariant pro- $\mathcal{C}$ subtree of $T$. By (a), $B \subseteq D$. Let $h \in H$; then $h B$ is also a minimal $N$-invariant pro- $\mathcal{C}$ subtree of $T$; hence $h B=B$. So $B$ is $H$-invariant. Therefore, by the minimality of $D, B=D$, as required.

Lemma 1.3. Let $H$ be an infinite abstract group that acts on an abstract tree $T$ so that the stabilizer of each vertex is finite. Then $T$ cannot contain disjoint $H$-subtrees. Consequently, if $T$ has a minimal $H$-invariant subtree, it is unique.

Proof. Let $T_{1}$ and $T_{2}$ be disjoint $H$-subtrees of $T$. Consider the graph $\tilde{T}$ obtained from $T$ by collapsing $T_{1}$ and $T_{2}$ to distinct vertices $v_{1}$ and $v_{2}$, respectively. Then $\tilde{T}$ is a tree (cf. Part I, Corollary 2 to Proposition 13 in [16]) on which $H$ acts with finite vertex stabilizers except for the vertices $v_{1}$ and $v_{2}$, which are fixed by $H$. Let $p$ be a path joining $v_{1}$ and $v_{2}$ in $\tilde{T}$. It follows that one can choose $h \in H$ so that $p$ and $h p$ are different paths joining $v_{1}$ and $v_{2}$ in $\tilde{T}$. One gets a contradiction with the fact that $\tilde{T}$ is a tree by observing that $p \cup h p$ contains a nontrivial cycle.

Consider now the following situation. Let $H$ be an abstract group which is embedded as a dense subgroup in an infinite pro- $\mathcal{C}$ group $\tilde{H}$. Assume that $T^{\text {abs }}$ is an abstract tree which is embedded as a dense subgraph of a pro- $\mathcal{C}$ tree $T$. We assume further that $\tilde{H}$ acts continuously on the pro- $\mathcal{C}$ tree $T$ in such a way that $T^{\text {abs }}$ is $H$ invariant and such that $H \backslash T^{\mathrm{abs}}$ is a finite graph, and suppose that the $H$-stabilizer of each vertex is finite (in reality we only need some condition to be certain of uniqueness of minimal invariant subtrees in case they exist for the abstract case; according to Lemma 1.3, this is guaranteed by the above condition).

Lemma 1.4. Assume in addition that the natural epimorphism of graphs

$$
H \backslash T^{\mathrm{abs}} \longrightarrow \tilde{H} \backslash T
$$

is an isomorphism. Then there exists a unique minimal $H$-invariant subtree $D^{\text {abs }}$ of $T^{\text {abs }}$ and its closure $D=\overline{D^{\text {abs }}}$ in $T$ is the unique minimal $\tilde{H}$-invariant pro-C subtree of $T$; moreover $D^{\text {abs }}=T^{\text {abs }} \cap D$ and $H \backslash D^{\text {abs }}=\tilde{H} \backslash D$ is finite.

Proof. We shall identify $H \backslash T^{\text {abs }}$ with $\tilde{H} \backslash T$. Consider the commutative diagram: 


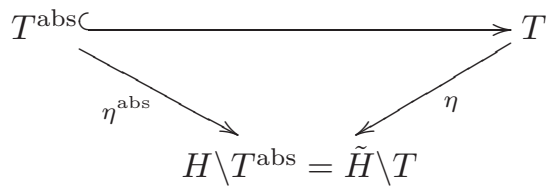

where $\eta^{\text {abs }}$ and $\eta$ are the canonical quotient maps of graphs. We observe that this means that if $x, y \in T^{\text {abs }}$ and $x \in \tilde{H} y$, then $x \in H y$. The $H$-invariant subgraphs of $T^{\text {abs }}$ have the form $\left(\eta^{\text {abs }}\right)^{-1}(R)$ for some subgraph $R$ of $H \backslash T^{\text {abs }}$, and, similarly, $\tilde{H}$-invariant subgraphs of $T$ have the form $\eta^{-1}(R)$. Since $H \backslash T$ abs is a finite graph, we deduce that $T^{\text {abs }}$ has a minimal $H$-invariant subgraph, which is unique by Lemma 1.3. We remark that if $T^{\prime}$ is an $\tilde{H}$-invariant pro- $\mathcal{C}$ subtree of $T$, then $T^{\text {abs }} \cap T^{\prime}$ is obviously $H$-invariant, and $T^{\text {abs }} \cap T^{\prime} \neq \emptyset$ (for $\left(\eta^{\text {abs }}\right)^{-1}\left(\eta\left(T^{\prime}\right)\right)$ $\subseteq T^{\text {abs }} \cap T^{\prime}$ ), so that $T^{\text {abs }} \cap T^{\prime}$ is a subtree of $T^{\text {abs }}$ (for if $v$ and $w$ are vertices of $T^{\text {abs }} \cap T^{\prime}$, then $[v, w] \subseteq T^{\prime}$ and also $\left.[v, w] \subseteq T^{\mathrm{abs}}\right)$. Furthermore, $\overline{T^{\text {abs }} \cap T^{\prime}}=T^{\prime}$. Indeed, let $\Sigma^{\prime}$ be a 0 -transversal of $\eta\left(T^{\prime}\right)$ in $T^{\text {abs }}$ with respect to the $H$-action (i.e., an $H$-transversal with $d_{0}(m) \in \Sigma^{\prime}$, for each $\left.m \in \Sigma^{\prime}\right)$. Then, by our hypothesis, $\Sigma^{\prime}$ is also a 0-transversal of $\eta\left(T^{\prime}\right)$ in $T$ with respect to the $\tilde{H}$-action. Hence $T^{\text {abs }} \cap T^{\prime}=$ $H \Sigma^{\prime}$ and $T^{\prime}=\tilde{H} \Sigma^{\prime}$, and in particular $\overline{T^{\text {abs }} \cap T^{\prime}}=T^{\prime}$.

Let $D$ be the unique $\tilde{H}$-invariant pro- $\mathcal{C}$ subtree of $T$ (see Lemma 1.2). Put $D^{\text {abs }}=T^{\text {abs }} \cap D$. We claim that $D^{\text {abs }}$ is the unique minimal $H$-invariant subtree of $T^{\text {abs }}$. Indeed, if $\Delta^{\text {abs }}$ were a proper $H$-invariant subtree of $D^{\text {abs }}$, we would be able to choose 0 -transversals $\Sigma^{\prime \prime}$ and $\Sigma^{\prime}$ of $\eta^{\text {abs }}\left(\Delta^{\text {abs }}\right)$ and $\eta^{\text {abs }}\left(D^{\text {abs }}\right)$, respectively, under the action of $H$, so that $\Sigma^{\prime \prime} \subset \Sigma^{\prime}$. Then by the above remark and the above observation, $\tilde{H} \Sigma^{\prime \prime}$ would be a proper $\tilde{H}$-invariant subtree of $D$, contradicting the minimality of $D$. This, together with Lemma 1.3, proves the claim.

Finally, since $H \backslash D^{\text {abs }} \subseteq H \backslash T$ abs and $\tilde{H} \backslash D \subseteq \tilde{H} \backslash T$, we deduce that $H \backslash D^{\text {abs }}=$ $\tilde{H} \backslash D$ and this graph is finite.

We apply this result to trees that arise as standard graphs (or covering graphs) of certain graphs of groups.

\section{Trees associated with virtually free groups}

Let $R$ be a finitely generated abstract free-by- $\mathcal{C}$ group. We explain next the construction of an abstract tree $S^{\text {abs }}$ and a pro-C tree $S$ associated with $R$ and with the pro-C completion $R_{\hat{\mathcal{C}}}$ of $R$, respectively, so that $S^{\text {abs }}$ is a dense subgraph of $S$.

Lemma 1.5. Let $R$ be a finitely generated abstract free-by-C group. Then:

(a) $R$ is the abstract fundamental group $\Pi^{\mathrm{abs}}=\Pi_{1}^{\mathrm{abs}}(\mathcal{G}, \Delta)$ of a graph of groups $(\mathcal{G}, \Delta)$ over a finite graph $\Delta$ such that each $\mathcal{G}(m)(m \in \Delta)$ is a finite group in $\mathcal{C}$;

(b) the pro-C fundamental group $\Pi=\Pi_{1}(\mathcal{G}, \Delta)$ of $(\mathcal{G}, \Delta)$ is the pro-C completion $R_{\hat{\mathcal{C}}}$ of $R$;

(c) in this case the canonical homomorphisms $\nu_{m}: \mathcal{G}(m) \longrightarrow \Pi(m \in \Delta)$ are embeddings. 
Proof. To fix the notation, let $\Phi$ be a normal free subgroup of $R$ such that $R / \Phi \in \mathcal{C}$.

(a) According to a result of Karrass-Pietrowski-Solitar (cf. Theorem 1 in [7]), $R$ is the abstract fundamental group $\Pi^{\text {abs }}=\Pi_{1}^{\text {abs }}(\mathcal{G}, \Delta)$ of a graph of groups $(\mathcal{G}, \Delta)$ over a finite graph $\Delta$ such that each $\mathcal{G}(m)(m \in \Delta)$ is a finite group. The isomorphic image $\Pi^{\text {abs }}(m)$ of $\mathcal{G}(m)(m \in \Delta)$ is a subgroup of $R=\Pi^{\text {abs }}$. On the other hand, a finite subgroup of $R$ is isomorphic to a subgroup of $R / \Phi$, and so it is in $\mathcal{C}$.

(b) Let $\nu_{m}: \mathcal{G}(m) \longrightarrow \Pi^{\text {abs }}=R$ be the inclusion $(m \in \Delta)$. Then $\Pi^{\text {abs }}$ together with the maps $\nu_{m}$ and a map $E(\Delta) \longrightarrow \Pi^{\text {abs }}$, written $e \mapsto t_{e}$, satisfies the universal property described in Section 0. By Lemma $0.4, R$ is residually $\mathcal{C}$ so that $R \leq R_{\hat{\mathcal{C}}}$. By abuse of notation we also write $\nu_{m}: \mathcal{G}(m) \longrightarrow R_{\hat{\mathcal{C}}}$ and $e \mapsto t_{e}$ for the compositions $\mathcal{G}(m) \hookrightarrow R \hookrightarrow R_{\hat{\mathcal{C}}}$ and $E(\Delta) \longrightarrow R \hookrightarrow R_{\hat{\mathcal{C}}}$. We shall show that $R_{\hat{\mathcal{C}}}$ together with these maps satisfies the universal property in the category of pro- $\mathcal{C}$ groups that characterizes the pro- $\mathcal{C}$ fundamental group $\Pi=\Pi_{1}^{\mathcal{C}}(\mathcal{G}, \Delta)$. Let $H$ be a pro-C $\mathcal{C}$ group and let $\beta_{m}: \mathcal{G}(m) \longrightarrow H(m \in \Delta)$ be homomorphisms and $e \mapsto s_{e}$ a map $E(\Delta) \longrightarrow H$ satisfying the condition (0.1) in Section 0. Since $R=\Pi^{\text {abs }}$, there exists a unique homomorphism $\delta: R \longrightarrow H$ with $\delta \nu_{m}=\beta_{m}(m \in \Delta)$ and $\delta\left(t_{e}\right)=s_{e}(e \in E(\Delta))$. Put $H_{1}=\operatorname{Im}(\delta)$. Let $U$ be an open normal subgroup of $H$. Then $R / \delta^{-1}(U) \cong H_{1} / H_{1} \cap U \leq H / U \in \mathcal{C}$, so that $R / \delta^{-1}(U) \in \mathcal{C}$. Hence $\delta^{-1}(U)$ is open in the pro- $\mathcal{C}$ topology of $R$, i.e., $\delta: R \longrightarrow H$ is continuous. Therefore $\delta$ extends uniquely to a continuous homomorphism $\tilde{\delta}: R_{\hat{\mathcal{C}}} \longrightarrow H$. Obviously $\tilde{\delta} \nu_{m}=\beta_{m}(m \in \Delta)$ and $\tilde{\delta}\left(t_{e}\right)=s_{e}(e \in E(\Delta))$; moreover, $\tilde{\delta}$ is the unique continuous homomorphism $R_{\hat{\mathcal{C}}} \longrightarrow H$ satisfying these conditions, for $\tilde{\delta}$ is determined by its restriction to the dense subgroup $R$ of $R_{\hat{\mathcal{C}}}$.

(c) This follows trivially from (a) and (b).

We continue with the assumptions and the notation of this lemma. Since in the present situation $\mathcal{G}(m)$ is finite, we have $\mathcal{G}(m) \cong \Pi^{\text {abs }}(m)=\Pi(m)(m \in \Delta)$. In particular, $\Pi^{\mathrm{abs}}(m)$ is closed in the pro- $\mathcal{C}$ topology of $R$. This together with the fact that $R=\Pi^{\text {abs }} \leq_{\text {dense }} R_{\hat{\mathcal{C}}}=\Pi$ implies that the natural map of graphs

$$
S^{\mathrm{abs}} \longrightarrow S
$$

given by $g \Pi^{\text {abs }}(m) \mapsto g \Pi(m)(m \in \Delta)$, is a dense embedding of the abstract tree $S^{\text {abs }}$ in the pro- $\mathcal{C}$ tree $S$ (see Proposition 0.2).

Proposition 1.6. Let $H=\left\langle h_{1}, \ldots, h_{r}\right\rangle$ be an infinite subgroup of $R=\Pi^{\text {abs }}$ that is finitely generated and closed in the pro-C topology of $R$, and let $\bar{H}$ be its closure in the pro-C group $R_{\hat{\mathcal{C}}}=\Pi$. Then $S^{\text {abs }}$ has a unique minimal $H$-invariant subtree $D^{\text {abs }}$, and its closure $D$ in $S$ is the unique minimal $\bar{H}$-invariant pro-C subtree of $S$. Furthermore, $S^{\text {abs }} \cap D=D^{\text {abs }}, \overline{D^{\text {abs }}}=D$, and $H \backslash D^{\text {abs }}=\bar{H} \backslash D$ is finite.

Proof. Choose a vertex $v_{0}$ of $\Delta$, and denote by $\tilde{v}_{0}$ the vertex $\tilde{v}_{0}=1 \Pi^{\operatorname{abs}}\left(v_{0}\right)=$ $1 \Pi\left(v_{0}\right)$ in $S^{\text {abs }} \subseteq S$. Define a subgraph $T^{\text {abs }}$ of $S^{\text {abs }}$ by

$$
T^{\mathrm{abs}}=\bigcup_{i=1}^{r} H\left[\tilde{v}_{0}, h_{i} \tilde{v}_{0}\right] .
$$


Let $L=\bigcup_{i=1}^{r}\left[\tilde{v}_{0}, h_{i} \tilde{v}_{0}\right]$. This is obviously a finite connected graph. Then $T^{\mathrm{abs}}=$ $H L$. Since $L \cap h_{i} L \neq \emptyset(i=1, \ldots, r)$, we have that $T^{\text {abs }}$ is a connected subgraph of the tree $S^{\text {abs }}$, and so $T^{\text {abs }}$ is a tree. Clearly it is $H$-invariant. Hence its closure in $S$,

$$
T=\overline{T^{\mathrm{abs}}}=\bigcup_{i=1}^{r} \bar{H}\left[\tilde{v}_{0}, h_{i} \tilde{v}_{0}\right]
$$

is a pro- $\mathcal{C}$ subtree of $S$; clearly it is $\bar{H}$-invariant.

Since $H$ is infinite and each $\mathcal{G}(m)$ is finite, our result will follow from Lemma 1.4 after we show that the epimorphism of graphs $H \backslash T^{\mathrm{abs}} \longrightarrow \bar{H} \backslash T$ is in fact an isomorphism. To see this we distinguish two cases.

Case 1. Assume that $H \leq \Phi$.

Since the $R$-stabilizers of the elements of $S^{\text {abs }}$ are finite groups, we have that $\Phi$ acts freely on $S^{\text {abs }}$. By Lemma 1.1, there exist an open subgroup $V$ of $\Phi$ (and so of $\Pi^{\text {abs }}$ ) containing $H$ such that the map of graphs

$$
H \backslash T^{\mathrm{abs}} \longrightarrow H \backslash S^{\mathrm{abs}} \longrightarrow V \backslash S^{\mathrm{abs}}
$$

is injective. Next observe that for every $m \in \Delta$, we have the following equality of double cosets:

$$
V \backslash \Pi^{\mathrm{abs}} / \Pi^{\mathrm{abs}}(m)=\bar{V} \backslash \Pi / \Pi(m)
$$

because $\Pi^{\text {abs }}(m)=\Pi(m), V$ has finite index in $\Pi^{\text {abs }}$, and $\Pi$ is the pro- $\mathcal{C}$ completion of $\Pi^{\text {abs }}$. Hence, one deduces that $V \backslash S^{\text {abs }}=\bar{V} \backslash S$ from the definitions of $S^{\text {abs }}$ and $S$ (see Section 0). Therefore, from the commutative diagram

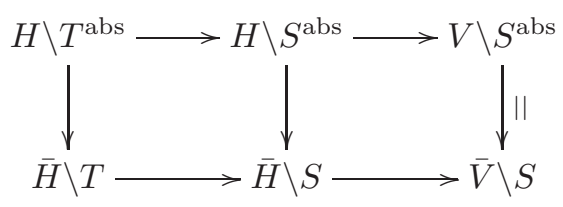

we deduce that $H \backslash T^{\mathrm{abs}}=\bar{H} \backslash T$, proving the result in this case.

Case 2. General case.

Define $K=\Phi \cap H$. Note that $K$ is closed in $\Phi$ and that $K \backslash T^{\text {abs }}$ is finite (because $K$ has finite index in $H$ ). So Lemma 1.1 can be used. Mimicking the argument in Case 1 one shows that $K \backslash T^{\mathrm{abs}}=\bar{K} \backslash T$. What this says is that if $t, t^{\prime} \in T^{\mathrm{abs}}$, and $\bar{K} t=\bar{K} t^{\prime}$, then $K t=K t^{\prime}$.

Now since $K$ has finite index in $H$, we have finite unions $H=\bigcup K x_{i}$ and $\bar{H}=\bigcup \bar{K} x_{i}$ (some $\left.x_{i} \in H\right)$. Let $t, t^{\prime} \in T^{\text {abs }}$, and assume that $\bar{H} t=\bar{H} t^{\prime}$. We want to show that $H t=H t^{\prime}$. By hypothesis we have $\bigcup \bar{K} x_{i} t=\bigcup \bar{K} x_{i} t^{\prime}$. So for each $i$, there are some $i^{\prime}$ and $i^{\prime \prime}$ such that $\bar{K} x_{i} t=\bar{K} x_{i^{\prime}} t^{\prime}$ and $\bar{K} x_{i} t^{\prime}=\bar{K} x_{i^{\prime \prime}} t$; hence $K x_{i} t=$ $K x_{i^{\prime}} t^{\prime}$ and $K x_{i} t^{\prime}=K x_{i^{\prime \prime}} t$. Therefore, $\bigcup K x_{i} t=\bigcup K x_{i} t^{\prime}$, i.e., $H t=H t^{\prime}$. 


\section{Standard tree of a finite graph of residually finite groups and the tits line}

Next we consider the case of a tree that arises as the standard graph of a more general graph of abstract groups $(\mathcal{G}, \Delta)$, but we shall restrict the type of the groups $H$ for which we seek minimal $H$-invariant subtrees and we only envision the profinite topology. We refer to Section 0 for the notation.

A subtree $L$ of an abstract tree is called a straight line if it is a doubly infinite chain of the form

Next we recall a result of J. Tits that we state in a manner convenient for us. We maintain the notation and the assumptions of Proposition 0.2. We say that an element $b \in \Pi^{\mathrm{abs}}$ is hyperbolic if it does not fix any vertex of the tree $S^{\text {abs }}$. If $v$ and $w$ are vertices of $S^{\text {abs }}$, we denote by $l(v, w)$ the length of the unique reduced path joining $v$ and $w$.

Lemma 1.7 (Proposition 24 in Part I of [16]). Let $b \in \Pi^{\text {abs }}$ be a hyperbolic element. Write

$$
m=\inf \left\{l(v, b v) \mid v \in V\left(S^{\mathrm{abs}}\right) \quad \text { and } \quad L_{b}=\left\{v \in V\left(S^{\mathrm{abs}}\right) \mid l(v, b v)=m\right\} .\right.
$$

Then

(a) $L_{b}$ is the set of vertices of a $\langle b\rangle$-invariant straight line (the 'Tits straight line corresponding to $\left.b^{\prime}\right)$ which we denote again by $L_{b}$; in fact the action of $b$ on $L_{b}$ is a translation with amplitude $m$;

(b) $L_{b}$ is contained in any $\langle b\rangle$-invariant subtree of $S^{\text {abs }}$; in fact $L_{b}$ is the unique minimal $\langle b\rangle$-invariant subtree of $S^{\text {abs }}$;

(c) if $v \in L_{b}$, then $L_{b}=\langle b\rangle[v, b v]$.

The aim of this subsection is to prove the following result.

Proposition 1.8. Let $\Delta$ be a finite connected graph and let $(\mathcal{G}, \Delta)$ be a graph of abstract groups over $\Delta$. Assume that its fundamental group $\Pi^{\text {abs }}=\Pi_{1}^{\text {abs }}(\mathcal{G}, \Delta)$ is residually finite and each $\Pi^{\mathrm{abs}}(m)$ is closed in the profinite topology of $\Pi^{\mathrm{abs}}$. As in Proposition 0.1 , let $(\overline{\mathcal{G}}, \Delta)$ be the graph of profinite groups over $\Delta$, where $\overline{\mathcal{G}}(m)$ is the completion of $\mathcal{G}(m)$ with respect to the topology induced from the profinite topology of $\Pi^{\mathrm{abs}}$. Let $\Pi=\Pi_{1}(\overline{\mathcal{G}}, \Delta)$ be the corresponding profinite fundamental group. Let $S^{\mathrm{abs}}$ (respectively, $S$ ) be the standard (respectively, standard profinite) tree of this graph of groups. Let $b \in \Pi^{\text {abs }}$ be a hyperbolic element of $\Pi^{\text {abs }}$ such that the subgroup $\langle b\rangle$ is closed in the profinite topology of $\Pi^{\mathrm{abs}}$, and let $L_{b}$ be the corresponding Tits straight line. Then

(a) $\langle b\rangle \backslash L_{b}=\overline{\langle b\rangle} \backslash \overline{L_{b}}$;

(b) $\overline{L_{b}}$ is the unique minimal $\overline{\langle b\rangle}$-invariant profinite subtree of $S$, and $\overline{L_{b}} \cap S$ abs $=L_{b}$. 
Before embarking on the proof of this proposition, we need two auxiliary results which are valid in more generality than is necessary for our purposes; for these results we consider the pro- $\mathcal{C}$ topology, where $\mathcal{C}$ is an extension-closed variety of finite groups. The first one (Proposition 1.9) was proved in [12], but we record it here for the convenience of the reader. The strategy in the proof of Proposition 1.8 above is to find a way to use Proposition 1.6; one cannot use it directly because it assumes that the fundamental group $\Pi^{\text {abs }}$ is free-by- $\mathcal{C}$. However, we show in our second auxiliary result (Proposition 1.10) that, under the hypotheses of the above proposition, $\Pi$ can be expressed as an inverse limit of pro- $\mathcal{C}$ fundamental groups which are the pro- $\mathcal{C}$ completions of abstract free-by- $\mathcal{C}$ groups.

Proposition 1.9. (Proposition 2.9 in [12]) Assume that the abstract fundamental group $\Pi^{\mathrm{abs}}=\Pi_{1}^{\mathrm{abs}}(\mathcal{G}, \Delta)$ of a graph of groups $(\mathcal{G}, \Delta)$ over a finite graph $\Delta$ is residually $\mathcal{C}$ and that $\Pi^{\text {abs }}(m)$ is closed in the pro- $\mathcal{C}$ topology of $\Pi_{1}^{\text {abs }}(\mathcal{G}, \Delta)$. Let $b \in \Pi^{\text {abs }}$ be hyperbolic. Then $\overline{\langle b\rangle}$ acts freely on the standard pro-C tree $S=S(\overline{\mathcal{G}}, \Delta)$ of the graph of pro-C groups $(\overline{\mathcal{G}}, \Delta)$.

Proposition 1.10. Let $(\mathcal{G}, \Delta)$ be a graph of abstract groups over a finite connected graph $\Delta$ such that $\Pi^{\mathrm{abs}}=\Pi_{1}^{\mathrm{abs}}(\mathcal{G}, \Delta)$ is residually $\mathcal{C}$ and each $\Pi^{\mathrm{abs}}(m)$ is closed in the pro-C topology of $\Pi_{1}^{\mathrm{abs}}(\mathcal{G}, \Delta)$. Let $\mathcal{U}$ be the collection of all open (in the pro-C topology) normal subgroups of $\Pi^{\mathrm{abs}}=\Pi_{1}^{\mathrm{abs}}(\mathcal{G}, \Delta)$. Then there is an inverse system of graphs of groups $\left(\mathcal{G}_{U}, \Delta\right)$ over $\Delta$ such that:

(a) Each $\mathcal{G}_{U}(m)$ is a group in $\mathcal{C}(m \in \Delta)$ and $(\overline{\mathcal{G}}, \Delta)={\underset{U}{U \in \mathcal{U}}}_{\lim }\left(\mathcal{G}_{U}, \Delta\right)$, where $(\overline{\mathcal{G}}, \Delta)$ is as in Proposition 0.2.

(b) For each $U \in \mathcal{U}$, let $\Pi_{U}=\Pi_{1}\left(\mathcal{G}_{U}, \Delta\right)$ be the pro-C fundamental group of the graph of groups $\left(\mathcal{G}_{U}, \Delta\right)$, and let $S_{U}=S\left(\mathcal{G}_{U}, \Delta\right)$ be the corresponding standard pro-C tree. Then

$$
\Pi={\underset{U \in \mathcal{U}}{\lim }}_{U} \Pi_{U} \quad \text { and } \quad S={\underset{U \in \mathcal{U}}{\lim }}_{S_{U}}
$$

where $\Pi$ is the fundamental pro-C group of $(\overline{\mathcal{G}}, \Delta)$, and where $S$ is the corresponding standard pro-C tree.

(c) For each $U \in \mathcal{U}$, the abstract fundamental group $\Pi_{U}^{\mathrm{abs}}=\Pi_{1}^{\mathrm{abs}}\left(\mathcal{G}_{U}, \Delta\right)$ of the graph of groups $\left(\mathcal{G}_{U}, \Delta\right)$ contains an open (in its pro-C topology) free subgroup, so that according to Lemma 1.5, $\Pi_{U}$ is the pro-C completion of $\Pi_{U}^{\mathrm{abs}}$, and the standard abstract tree $S_{U}^{\mathrm{abs}}$ corresponding to $\left(\mathcal{G}_{U}, \Delta\right)$ is densely embedded in $S_{U}$.

(d) The canonical projections $\varphi_{U}: \Pi \longrightarrow \Pi_{U}$ and $\psi_{U}: S \longrightarrow S_{U}$ are compatible with the actions of $\Pi$ on $S$ and of $\Pi_{U}$ on $S_{U}$, i.e., $\psi_{U}(g a)=\varphi_{U}(g) \psi_{U}(a)$, $(g \in \Pi, a \in S)$; furthermore, $\varphi_{U}\left(\Pi^{\mathrm{abs}}\right)=\Pi_{U}^{\mathrm{abs}}$ and $\psi_{U}\left(S^{\mathrm{abs}}\right)=S_{U}^{\mathrm{abs}}$, for each $U \in \mathcal{U}$.

(e) Let $H$ be a closed subgroup of $\Pi$ that acts freely on $S$. Then there exists some $\tilde{U} \in \mathcal{U}$ such that for all $V \in \mathcal{U}$ with $V \leq \tilde{U}$ one has that $\varphi_{V}(H)$ acts freely on $S_{V}$. 
Proof. For each $U \in \mathcal{U}$, consider the graph of groups $\left(\mathcal{G}_{U}, \Delta\right)$ over $\Delta$ with

$$
\mathcal{G}_{U}(m)=\Pi^{\mathrm{abs}}(m) / \Pi^{\mathrm{abs}}(m) \cap U \quad(m \in \Delta) .
$$

The only parts that require an explicit proof are (c) and (e), for (a), (b) and (d) are immediate consequences of the definitions.

(c) For $U \in \mathcal{U}$, denote by $\tilde{U}$ the subgroup of $\Pi^{\text {abs }}$ generated by the $U$-stabilizers of the vertices of $S^{\text {abs }}$, i.e.,

$$
\tilde{U}=\left\langle U \cap g \Pi^{\mathrm{abs}}(v) g^{-1} \mid v \in V(\Delta), g \in \Pi^{\mathrm{abs}}\right\rangle .
$$

Clearly $\tilde{U} \triangleleft \Pi^{\text {abs }}$. Then $\tilde{U} \backslash S^{\text {abs }}$ is a tree (this follows from [16], Corollary 1 to Theorem 13, page 55; explicitly, see Exercise 2 on that page). Now, $\Pi^{\text {abs }} / \tilde{U}$ acts on $\tilde{U} \backslash S^{\text {abs }}$ and

$$
\left(\Pi^{\mathrm{abs}} / \tilde{U}\right) \backslash\left(\tilde{U} \backslash S^{\mathrm{abs}}\right)=\Pi^{\mathrm{abs}} \backslash S^{\mathrm{abs}}=\Delta .
$$

Furthermore the $\Pi^{\text {abs }} / \tilde{U}$-stabilizer of the vertex $\tilde{U} 1 \Pi^{\text {abs }}(m)$ of $\tilde{U} \backslash S^{\text {abs }}$ is

$$
\tilde{U} \Pi^{\mathrm{abs}}(m) / \tilde{U} \cong \Pi^{\mathrm{abs}}(m) / \tilde{U} \cap \Pi^{\mathrm{abs}}(m)=\Pi^{\mathrm{abs}}(m) / U \cap \Pi^{\mathrm{abs}}(m) .
$$

Therefore,

$$
\Pi^{\mathrm{abs}} / \tilde{U}=\Pi_{1}^{\mathrm{abs}}\left(\mathcal{G}_{U}, \Delta\right)=\Pi_{U}^{\mathrm{abs}} \quad \text { and } \quad S_{U}^{\mathrm{abs}}=\tilde{U} \backslash S^{\mathrm{abs}}
$$

(cf. Theorem 13, page 55, in [16]). Finally observe that $U / \tilde{U}$ acts freely on the tree $\tilde{U} \backslash S^{\text {abs }}$, and hence it is free (cf. Theorem 4, page 27, in [16]). Obviously $U / \tilde{U}$ is open in the pro-C topology of $\Pi_{U}^{\text {abs }}$, since $\left(\Pi^{\text {abs }} / \tilde{U}\right) /(U / \tilde{U}) \cong \Pi^{\text {abs }} / U \in \mathcal{C}$.

(e) Let $Y$ (respectively, $Y_{U}$, where $U \in \mathcal{U}$ ) be the compact subspace of the points of $S$ (respectively, $S_{U}$ ) fixed by $H$ (respectively, $\varphi_{U}(H)$ ); clearly

$$
Y=\varliminf_{U \in \mathcal{U}}^{\lim } Y_{U}
$$

Since $H$ acts freely on $S, Y=\emptyset$. By compactness, there exists some $\tilde{U} \in \mathcal{U}$ such that $Y_{V}=\emptyset$ whenever $V \in \mathcal{U}$ and $V \leq \tilde{U}$ (cf. Proposition 1.1.4 in [14]). This means that $\varphi_{V}(H)$ acts freely on $S_{V}$ for all such $V$.

Proof of Proposition 1.8. We only need to prove part (a) (part (b) then follows from (a) and Lemma 1.4). Since $b$ is hyperbolic, it has infinite order. According to Proposition $1.9, \overline{\langle b\rangle}$ acts freely on the profinite tree $S=S(\overline{\mathcal{G}}, \Delta)$. We continue with the notation of Proposition 1.10. Let $b_{U}=\varphi_{U}(b)$ denote the image of $b$ in $\Pi_{U}$. By Proposition $1.10(\mathrm{e})$, there exists some $\tilde{U} \in \mathcal{U}$ such that $\overline{\left\langle b_{V}\right\rangle}$ acts freely on $S_{V}$ for every $V \leq \tilde{U}$. In particular we may assume that each $b_{V}$ has infinite order. We claim that $\overline{\left\langle b_{V}\right\rangle} \cong \widehat{\mathbf{Z}}$. To see this it suffices to show that the profinite topology of $\Pi_{V}^{\text {abs }}$ induces on $\left\langle b_{V}\right\rangle$ its full profinite topology. Let $F_{V}$ be an open (in the profinite topology) free subgroup of $\Pi_{V}^{\text {abs }}$ (see Proposition $1.10(\mathrm{c})$ ). Then $\Pi_{V}^{\mathrm{abs}}$ induces on $F_{V}$ its full profinite topology (cf. Lemma 3.1.4 in [14]). Now by a result of M. Hall, $F_{V} \cap\left\langle b_{V}\right\rangle$ is a free factor of a subgroup of finite index in $F_{V}$ (cf. Theorem 3.10 in 
Chapter I of [8]). Therefore the profinite topology of $F_{V}$ (and so of $\Pi_{V}^{\text {abs }}$ ) induces on $\left\langle b_{V}\right\rangle$ its full profinite topology (cf. Lemma 3.1.4 and Corollary 3.1.6 in [14]), proving the claim. One deduces that the natural epimorphism $\overline{\langle b\rangle} \longrightarrow \overline{\left\langle b_{V}\right\rangle}$ is an isomorphism.

By assumption $\Pi^{\text {abs }}(m)$ is closed in $\Pi^{\text {abs }}(m \in \Delta)$, and so we have $S^{\text {abs }} \subseteq S$ (see Proposition 0.2). Similarly, note that each $\Pi_{U}^{\mathrm{abs}}(m)$ is a finite group and so closed in the profinite topology of $\Pi_{U}^{\mathrm{abs}}$. By Proposition 1.10, $\Pi_{U}$ is the profinite completion of $\Pi_{U}^{\mathrm{abs}}$, and $S_{U}^{\mathrm{abs}} \subseteq S_{U}$. Moreover, $\varphi_{U}\left(\Pi^{\mathrm{abs}}\right)=\Pi_{U}^{\mathrm{abs}}$ and $\psi_{U}\left(S^{\mathrm{abs}}\right)=$ $S_{U}^{\mathrm{abs}}$. For every $U \in \mathcal{U}, \varphi_{U}\left(L_{b}\right)$ is connected and so is a subtree of the tree $S_{U}^{\mathrm{abs}}$ which is $\left\langle b_{U}\right\rangle$-invariant (see Part (d) of Proposition 1.10); therefore, $L_{b_{U}} \subseteq \varphi_{U}\left(L_{b}\right)$.

Choose a vertex $v \in V\left(L_{b}\right)$ of $L_{b}$. Since $[v, b v]$ is finite and since $S=\lim _{\longleftarrow} S_{U}$, there exists some $U_{0} \in \mathcal{U}$ such that the restriction of $\psi_{U}$ to $[v, b v]$ is an injection for every $U \leq U_{0}$. Choose $U^{\prime} \leq U_{0} \cap \tilde{U}$. Then $\psi_{U^{\prime}}$ sends $\overline{L_{b}}=\overline{\langle b\rangle}[v, b v]$ onto $\psi_{U^{\prime}}\left(\overline{L_{b}}\right)=\overline{\left\langle b_{U^{\prime}}\right\rangle}\left[\psi_{U^{\prime}}(v), b_{U^{\prime}} \psi_{U^{\prime}}(v)\right]$ bijectively and hence $\psi_{U^{\prime}}\left(\overline{L_{b}}\right)$ is minimal. Since $L_{b_{U^{\prime}}} \subseteq \varphi_{U^{\prime}}\left(\overline{L_{b}}\right)$, we deduce that $\psi_{U^{\prime}}$ sends the $\overline{\langle b\rangle}$-space $\overline{L_{b}}$ to the $\overline{\left\langle b_{U^{\prime}}\right\rangle}$ - space $\overline{L_{b_{U^{\prime}}}}$ isomorphically; similarly $\varphi_{U^{\prime}}\left(L_{b}\right)=L_{b_{U^{\prime}}}$. Since $\left\langle b_{U^{\prime}}\right\rangle$ is closed in the profinite topology of $\Pi_{U^{\prime}}^{\text {abs }}$, by Proposition 1.6, $\left\langle b_{U^{\prime}}\right\rangle \backslash L_{b_{U^{\prime}}}=\overline{\left\langle b_{U^{\prime}}\right\rangle} \backslash \overline{L_{b_{U^{\prime}}}}$. Hence $\langle b\rangle \backslash L_{b}=$ $\overline{\langle b\rangle} \backslash \overline{L_{b}}$. This completes the proof of (a).

\section{Closure of normalizers}

Let $R$ be an abstract group which is residually $\mathcal{C}$ and let $H$ be a finitely generated closed (in the pro- $\mathcal{C}$ topology of $R$ ) subgroup of $R$. In this section we study the relationship between the normalizer $N_{R}(H)=\left\{x \in R \mid x^{-1} H x=H\right\}$ of $H$ in $R$ and the normalizer $N_{R_{\hat{\mathcal{C}}}}(\bar{H})$ of $\bar{H}$ in $R_{\hat{\mathcal{C}}}$, where $\bar{H}$ is as usual the closure of $H$ in $R_{\hat{\mathcal{C}}}$. When $R$ is finitely generated and contains an open free abstract subgroup, we show (Theorem 2.6) that the first normalizer is dense in the latter. In particular this is the case if $R$ is free-by-finite and $H$ is any finitely generated subgroup, when $\mathcal{C}$ is the class of all finite groups (Corollary 2.9). The crucial point for the proof of this result is that we can use 'combinatorial' methods, in the form of groups acting on trees, and the interrelation between abstract and profinite groups and graphs that we have developed in Section 1, precisely for this type of group $R$.

Lemma 2.1. Let $R$ be an abstract group which is residually $\mathcal{C}$. Let $H$ and $K$ be subgroups of $R$, which are closed in the pro-C topology of $R$. Then

$$
R \cap N_{\bar{K}}(\bar{H})=N_{K}(H) .
$$

Proof. First we claim that $N_{K}(H)=N_{K}(\bar{H})$. Clearly $N_{K}(H) \leq N_{K}(\bar{H})$. Conversely, let $k \in N_{K}(\bar{H})$; then if $h \in H$, we have $k^{-1} h k \in \bar{H} \cap R=H$, since $H$ is closed in the profinite topology of $R$; therefore $k \in N_{K}(H)$. This proves the claim. Since $K$ is also closed in the pro- $\mathcal{C}$ topology of $R$, we have $R \cap \bar{K}=K$. It follows that $R \cap N_{\bar{K}}(\bar{H})=N_{K}(\bar{H})=N_{K}(H)$. 
Lemma 2.2. Let $C \in \mathcal{C}$ be a group of prime order $p$. Let $R=\Phi \rtimes C$ be a semidirect product, where $\Phi$ is a finitely generated abstract free group. Let $H$ be a subgroup of $R$ of order $p$. Then there is a free factor $\Phi_{1}$ of $\Phi$ such that

(a) $N_{R}(H)=H \times \Phi_{1}$ and $N_{R_{\hat{\mathcal{C}}}}(\bar{H})=H \times\left(\Phi_{1}\right)_{\hat{\mathcal{C}}}$,

and

(b) $C_{\Phi}(H)=\Phi_{1}$ and $C_{\Phi_{\hat{\mathcal{C}}}}(H)=\left(\Phi_{1}\right)_{\hat{\mathcal{C}}}$, where $C_{\Phi}(H)=\{x \in \Phi \mid x h=h x, \forall h \in H\}$ is the centralizer of $H$ in $\Phi$.

Consequently,

$\left(\mathrm{a}^{\prime}\right) \overline{N_{R}(H)}=N_{R_{\hat{\mathcal{C}}}}(H)$;

$\left(\mathrm{b}^{\prime}\right) \overline{C_{\Phi}(H)}=C_{\bar{\Phi}}(H)$.

Proof. By a theorem of Dyer-Scott (cf. Theorem 1 in [3]) the group $R$ is a free product,

$$
R=\left[*_{i=1}^{n}\left(C_{i} \times \Phi_{i}\right)\right] * L,
$$

where $L$ and each $\Phi_{i}$ are free groups and the $C_{i}$ are groups of order $p$. One deduces that

$$
R_{\hat{\mathcal{C}}}=\left[\coprod_{i=1}^{n}\left(C_{i} \times\left(\Phi_{i}\right)_{\hat{\mathcal{C}}}\right)\right] \amalg L_{\hat{\mathcal{C}}} .
$$

Since every finite subgroup of $R$ of order $p$ is conjugate to one of the $C_{i}$, we may assume without loss of generality that $H=C_{1}$ is in the first factor. Then $N_{R}(H)=$ $H \times \Phi_{1}$ and $N_{R_{\hat{\mathcal{C}}}}(\bar{H})=H \times\left(\Phi_{1}\right)_{\hat{\mathcal{C}}}(\mathrm{cf}$. Theorem B' in [6] or Theorem 9.1.12 in [14]), which is its closure. Observe that each $\Phi_{i}$ is a subgroup of $\Phi$ because $C_{i} \times N_{\Phi}\left(C_{i}\right)=N_{R}\left(C_{i}\right)=\mathcal{C}_{R}\left(C_{i}\right)=C_{i} \times \Phi_{i}$ (cf. Corollary 4.1 .5 in [9]) so that $\mathcal{C}_{R}\left(C_{i}\right)$ is finitely generated. Notice that $C_{\Phi}(H)=C_{R}(H) \cap \Phi=\left(H \times \Phi_{1}\right) \cap \Phi=\Phi_{1}$, since $\Phi_{1} \leq \Phi$, and similarly $C_{\Phi_{\hat{\mathcal{C}}}}(H)=\left(\Phi_{1}\right)_{\hat{\mathcal{C}}}$. Now, $C_{\Phi}(H)$ is the subgroup of fixed points of $\Phi$ under the action of $H$, and so $C_{\Phi}(H)=\Phi_{1}$ is a free factor of $\Phi$ (cf. Theorem 2 in [3]). This implies that $\left(\Phi_{1}\right)_{\hat{\mathcal{C}}}=\overline{\Phi_{1}}$ (cf. Corollary 3.1.6 in [14]). This concludes the proof of the lemma.

Recall (see Lemma 0.4) that if $R$ is an abstract group which contains a normal free subgroup $\Phi$ with $R / \Phi \in \mathcal{C}$, then $R$ is residually $\mathcal{C}$, and in particular $R \leq R_{\hat{\mathcal{C}}}$.

Proposition 2.3. Let $R$ be a finitely generated abstract free-by-C group, endowed with the pro-C topology. Let $H_{1}$ and $H_{2}$ be finitely generated closed subgroups of $R$. Then

$$
\overline{H_{1} \cap H_{2}}=\overline{H_{1}} \cap \overline{H_{2}} \quad \text { in } R_{\hat{\mathcal{C}}} .
$$

Proof. Obviously $\overline{H_{1} \cap H_{2}} \leq \overline{H_{1}} \cap \overline{H_{2}}$, so it is enough to prove that

$$
\overline{H_{1}} \cap \overline{H_{2}} \leq \overline{H_{1} \cap H_{2}} .
$$

Say $\Phi^{\prime}$ is an open free group subgroup of $R$. By Corollary 3.3 in [11], there exists an open subgroup $\Phi$ of $\Phi^{\prime}$ such that $\Phi=\left(H_{1} \cap \Phi^{\prime}\right) * M=\left(H_{1} \cap \Phi\right) * M$, for 
some subgroup $M$ of $\Phi$. Note that the pro- $\mathcal{C}$ topology of the clopen subgroup $\Phi$ coincides with the topology induced from the pro- $\mathcal{C}$ topology of $R$ (cf. Lemma 3.1.4 in [14]), so that $\bar{\Phi}=\Phi_{\hat{\mathcal{C}}} \leq R_{\hat{\mathcal{C}}}$. Hence, if $X \subseteq \Phi$, the notation $\bar{X}$ is unambiguous: it represents both the closure in $\Phi_{\hat{\mathcal{C}}}$ or in $R_{\hat{\mathcal{C}}}$.

We claim that to prove (2.1) it suffices to prove that

$$
\left(\overline{H_{1} \cap \Phi}\right) \cap\left(\overline{H_{2} \cap \Phi}\right)=\overline{H_{1} \cap H_{2} \cap \Phi} .
$$

Indeed, assume that (2.2) holds. Since $H_{i} \cap \Phi$ is open in $H_{i}$, one has a finite union $H_{i}=\bigcup_{j=1}^{s_{i}}\left(H_{i} \cap \Phi\right) g_{j}^{(i)}$, where $g_{1}^{(i)}, \ldots, g_{s_{i}}^{(i)} \in H_{i}$. Therefore $\overline{H_{i}}=\bigcup_{j=1}^{s_{i}}\left(\overline{H_{i} \cap \Phi}\right) g_{j}^{(i)}$ $(i=1,2)$. To prove $(2.1)$, let $u \in \overline{H_{1}} \cap \overline{H_{2}}$. Then $u=a_{1} h_{1}=a_{2} h_{2}\left(a_{i} \in \overline{H_{i} \cap \Phi}\right.$, $\left.h_{i} \in H_{i}\right)(i=1,2)$. So,

$$
a_{1}^{-1} a_{2}=h_{1} h_{2}^{-1} \in\left(\overline{H_{1} \cap \Phi}\right)\left(\overline{H_{2} \cap \Phi}\right) \cap R .
$$

Observe that $\left(\overline{H_{1} \cap \Phi}\right)\left(\overline{H_{2} \cap \Phi}\right)=\overline{\left(H_{1} \cap \Phi\right)\left(H_{2} \cap \Phi\right)}$. By Theorem 5.1 in [11], $\left(H_{1} \cap \Phi\right)\left(H_{2} \cap \Phi\right)$ is a closed subset in the pro- $\mathcal{C}$ topology of $\Phi$, so $\left(\overline{H_{1} \cap \Phi}\right)\left(\overline{H_{2} \cap \Phi}\right) \cap$ $R=\left(H_{1} \cap \Phi\right)\left(H_{2} \cap \Phi\right)$. We deduce that $a_{1}^{-1} a_{2}=h_{1} h_{2}^{-1}=b_{1}^{-1} b_{2}$, where $b_{i} \in H_{i} \cap \Phi$ $(i=1,2)$. Therefore, $b_{1} h_{1}=b_{2} h_{2} \in H_{1} \cap H_{2}$. Also, using assumption (2.2), $v=b_{1} a_{1}^{-1}=b_{2} a_{2}^{-1} \in\left(\overline{H_{1} \cap R}\right) \cap\left(\overline{H_{2} \cap R}\right)=\overline{H_{1} \cap H_{2} \cap R}$. Thus,

$$
u=a_{1} h_{1}=v^{-1} b_{1} h_{1} \in \overline{H_{1} \cap H_{2}},
$$

proving the claim.

It remains to prove (2.2). To simplify the notation we shall restate $(2.2)$ in the following manner: assume that $\Phi$ is a free abstract group of finite rank, $H_{1}$ and $H_{2}$ are closed finitely generated subgroups of $\Phi$, and $\Phi=H_{1} * M$, where $M$ is a subgroup of $\Phi$; then (2.2) says $\overline{H_{1}} \cap \overline{H_{2}}=\overline{H_{1} \cap H_{2}}$. We shall prove this.

Let $H=H_{1} \cap H_{2}$. Note that $H$ is also finitely generated by Howson's theorem (cf. [8], page 18). We need to prove

$$
\bar{H}=\overline{H_{1}} \cap \overline{H_{2}} \text {. }
$$

To do this we embed $\Phi$ in an appropriately chosen larger group $L$ which we construct as follows: consider an isomorphic copy $\Phi^{\prime}$ of $\Phi$ under an isomorphism

$$
\rho: \Phi \longrightarrow \Phi^{\prime}
$$

If $a$ is an element (respectively, a subset) of $\Phi$, we denote by $a^{\prime}$ the corresponding element $\rho(a)$ (respectively, subset) of $\Phi^{\prime}$ under that isomorphism. Furthermore, we assume that this isomorphism is the identity on $H_{1}$, i.e., it identifies $H_{1}$ with $H_{1}^{\prime}$, so that $\Phi \cup \Phi^{\prime}$ is an amalgam of groups with $\Phi \cap \Phi^{\prime}=H_{1}$. Let

$$
L=\Phi *_{H_{1}} \Phi^{\prime}
$$

be the amalgamated product of the groups $\Phi$ and $\Phi^{\prime}$ amalgamating $H_{1}$. Obviously, $L$ is a free group of finite rank. In fact,

$$
L=M * H_{1} * M^{\prime}=\Phi * M^{\prime}=M * \Phi^{\prime} .
$$


By the Kurosh subgroup theorem for subgroups of free products (cf. for example [8]) applied to (2.3) we have

$$
H_{2}=\left(H_{1} \cap H_{2}\right) * A \text { and } H_{2}^{\prime}=\left(H_{1} \cap H_{2}\right) * A^{\prime},
$$

where $A$ is a subgroup of $\mathrm{H}_{2}$.

Observe that the subgroups $\Phi, \Phi^{\prime}, H_{1}, H_{2}, H_{2}^{\prime}, H=H_{1} \cap H_{2}=H^{\prime}, A$ and $A^{\prime}$ are all finitely generated. Furthermore, they are closed in the pro- $\mathcal{C}$ topology of $L$ and their pro- $\mathcal{C}$ topologies coincide with the topologies induced from the pro- $\mathcal{C}$ topology of $L$. Indeed, $\Phi, \Phi^{\prime}$ and $H_{1}$ are free factors of $L$, and so for these groups the statements follow from Corollary 3.1.6 in [14]. In the case of $H_{2}$ and $H$, we know that these subgroups are closed in $R$, and so for these groups the statements follow from Corollary 3.3 in [11]; the argument is similar for $H_{2}^{\prime}$; finally $A$ and $A^{\prime}$ are closed since they are free factors of $H_{2}$ and $H_{2}^{\prime}$, respectively, and so closed. In particular the notation $\bar{\Phi}, \overline{H_{1}}, \overline{H_{2}}$, etc., is unambiguous: it has the same meaning whether these closures are taken in $L_{\hat{\mathcal{C}}}, \Phi_{\hat{\mathcal{C}}}$, etc. Hence, from now on, closures are assumed to be taken in $L_{\hat{\mathcal{C}}}$, and they coincide with their own pro-C completions: $\overline{H_{2}}=\left(H_{2}\right)_{\hat{\mathcal{C}}}$, etc. Therefore we have

$$
L_{\hat{\mathcal{C}}}=\Phi_{\hat{\mathcal{C}}} \amalg_{\left(H_{1}\right)_{\hat{\mathcal{C}}}} \Phi_{\hat{\mathcal{C}}}^{\prime}=\bar{\Phi} \amalg_{\overline{H_{1}}} \overline{\Phi^{\prime}},
$$

the pro- $\mathcal{C}$ amalgamated product, and

$$
L_{\hat{\mathcal{C}}}=\bar{M} \amalg \overline{H_{1}} \amalg \overline{M^{\prime}}=\bar{\Phi} \amalg \overline{M^{\prime}}=\bar{M} \amalg \overline{\Phi^{\prime}}
$$

(here $\amalg$ stands for free pro- $\mathcal{C}$ product).

Consider the subgroup $P=\left\langle H_{2}, H_{2}^{\prime}\right\rangle$ of $L$ generated by $H_{2}$ and $H_{2}^{\prime}$. Then

$$
P=\left\langle H_{2}, H_{2}^{\prime}\right\rangle=H_{2} *_{H_{1} \cap H_{2}} H_{2}^{\prime}=A *\left(H_{1} \cap H_{2}\right) * A^{\prime} .
$$

Next we assert that

$$
\bar{P} \cap \bar{\Phi}=\overline{H_{2}} \quad \text { and } \quad \bar{P} \cap \bar{\Phi}^{\prime}=\overline{H_{2}^{\prime}}
$$

To see this define a continuous epimorphism $\varphi: L_{\hat{\mathcal{C}}} \longrightarrow \bar{\Phi}$ by sending $\bar{\Phi}$ to $\bar{\Phi}$ identically, and $\bar{\Phi}^{\prime}$ to $\bar{\Phi}$ by means of $\rho^{-1}$. Note that $\varphi(\bar{P})=\overline{H_{2}}$. The assertions follow. We deduce that

$$
\bar{P} \cap \overline{H_{1}}=\bar{P} \cap \overline{H_{1}} \cap \overline{H_{1}}=\bar{P} \cap \bar{\Phi} \cap \overline{\Phi^{\prime}} \cap \overline{H_{1}}=\overline{H_{2}} \cap \overline{H_{2}^{\prime}} \cap \overline{H_{1}}=\overline{H_{2}} \cap \overline{H_{1}}
$$

(the last equality holds since, by the definition of $\Phi^{\prime}$, one has $\overline{H_{2}} \cap \overline{H_{1}}=\overline{H_{2}^{\prime}} \cap \overline{H_{1}}$ ).

We claim that $P$ is closed in the pro- $\mathcal{C}$ topology of $L$, i.e., that

$$
P=L \cap \bar{P} \text {. }
$$

To prove this we use the standard tree $S^{\text {abs }}$ associated with the amalgamated product $L=\Phi *_{H_{1}} \Phi^{\prime}$, and the standard pro- $\mathcal{C}$ tree $S$ associated with the amalgamated pro- $\mathcal{C}$ product $L_{\hat{\mathcal{C}}}=\bar{\Phi} \amalg_{\overline{H_{1}}} \overline{\Phi^{\prime}}$ which we described in Section 0 . In these 
cases $L=\Phi *_{H_{1}} \Phi^{\prime}$ and $L_{\hat{\mathcal{C}}}=\bar{\Phi} \amalg_{\overline{H_{1}}} \overline{\Phi^{\prime}}$ are the abstract and pro-C fundamental groups of graphs of groups over a graph $\Delta$ with a single edge and two different vertices. We recall the explicit definitions of $S^{\text {abs }}$ and $S$ in these specific situations: the vertices of $S^{\text {abs }}$ are the elements of $L / \Phi \cup L / \Phi^{\prime}$ and its set of edges is $L / H_{1}$; moreover, the origin of an edge $x H_{1}(x \in L)$ is $d_{0}\left(x H_{1}\right)=x \Phi$, and its terminal vertex is $d_{1}\left(x H_{1}\right)=x \Phi^{\prime}$. Similarly, the pro-C tree $S$ has vertices $L_{\hat{\mathcal{C}}} / \bar{\Phi} \bullet L_{\hat{\mathcal{C}}} / \overline{\Phi^{\prime}}$ and edges $L_{\hat{\mathcal{C}}} / \overline{H_{1}}$, with $d_{0}\left(x \overline{H_{1}}\right)=x \bar{\Phi}$ and $d_{1}\left(x \overline{H_{1}}\right)=x \overline{\Phi^{\prime}}$, where $x \in L_{\hat{\mathcal{C}}}$. Now, the map $S^{\text {abs }} \longrightarrow S$ given by $x \Phi \mapsto x \bar{\Phi}, x \Phi \mapsto x \overline{\Phi^{\prime}}$ and $x H_{1} \mapsto x \overline{H_{1}}(x \in L)$ is an embedding of graphs because by assumption the subgroups $\Phi, \Phi^{\prime}$ and $H_{1}$ are closed in the pro-C topology of $L$ (see Proposition 0.2). We think of $S^{\text {abs }}$ as being a dense subgraph of $S$. Denote by $e \in S^{\text {abs }} \subseteq S$ the edge $e=1 H_{1}=1 \overline{H_{1}}$, so that $S^{\mathrm{abs}}=L\left\{e, d_{0}(e), d_{1}(e)\right\}$ and $S=L_{\hat{\mathcal{C}}}\left\{e, d_{0}(e), d_{1}(e)\right\}$.

Choose $g \in L \cap \bar{P}$. To prove (2.4) we need to show that $g \in P$. Note that $g e \in \bar{P} e \subseteq S$. Denote by $[e, g e]$ the smallest pro- $\mathcal{C}$ subtree of $S$ containing $e$ and ge. Since $e, g e \in S^{\text {abs }},[e, g e]$ is a finite subtree of $S^{\text {abs }}$ : it is the subtree of $S^{\text {abs }}$ underlying the unique reduced path of $S^{\text {abs }}$ joining $e$ and ge. Note that $\overline{H_{2}}$ fixes $d_{0}(e)=1 \bar{\Phi}$ and $\overline{H_{2}^{\prime}}$ fixes $d_{1}(e)=1 \overline{\Phi^{\prime}}$. Since $\bar{P}$ is generated topologically by $\overline{H_{2}}$ and $\overline{H_{2}^{\prime}}$, and since the segment $\left\{e, d_{0}(e), d_{1}(e)\right\}$ is obviously connected, we deduce that $\bar{P}\left\{e, d_{0}(e), d_{1}(e)\right\}$ is a pro- $\mathcal{C}$ subtree of $S$. Therefore, $[e, g e] \subseteq \bar{P}\left\{e, d_{0}(e), d_{1}(e)\right\}$. Now, since $[e, g e]$ is finite, it consists of a finite sequence of edges

$$
e, \bar{p}_{1} e, \bar{p}_{1} \bar{p}_{2} e, \bar{p}_{1} \bar{p}_{2} \bar{p}_{3} e, \ldots, \bar{p}_{1} \bar{p}_{2} \cdots \bar{p}_{n} e=g e,
$$

where the elements $\bar{p}_{1}, \bar{p}_{2}, \ldots, \bar{p}_{n}$ belong to $\bar{\Phi}$ or $\overline{\Phi^{\prime}}$ (alternating). Since this path is also in $\bar{P}\left\{e, d_{0}(e), d_{1}(e)\right\}$, we have that $\bar{p}_{1}, \bar{p}_{1} \bar{p}_{2}, \bar{p}_{1} \bar{p}_{2} \bar{p}_{3}, \ldots \bar{p}_{1} \bar{p}_{2} \cdots \bar{p}_{n} \in \bar{P}$; hence one deduces inductively that $\bar{p}_{1}, \bar{p}_{2}, \ldots, \bar{p}_{n}$ are elements of either $\bar{P} \cap \bar{\Phi}=\overline{H_{2}}$ or of $\bar{P} \cap \overline{\Phi^{\prime}}=\overline{H_{2}^{\prime}}$. Furthermore $g=\bar{p}_{1} \bar{p}_{2} \cdots \bar{p}_{n} \bar{h}$, where $\bar{h} \in \overline{H_{1}}$, since the $L_{\hat{\mathcal{C}}^{\text {-stabilizer }}}$ of $e$ is $\overline{H_{1}}$.

Now, since the subgroups $H_{1}, H_{2}$ and $H_{2}^{\prime}$ are finitely generated and closed in the pro- $\mathcal{C}$ topology of the free abstract group $L$, we have that any finite product $K_{1} \cdots K_{n} H_{1}$, with $K_{i} \in\left\{H_{2}, H_{2}^{\prime}\right\}$, is closed (cf. Theorem 5.1 in [11]). Hence $K_{1} \cdots K_{n} H_{1}=L \cap\left(\overline{K_{1}} \cdots \overline{K_{n}} \overline{H_{1}}\right)$. Since $g \in L$, we deduce that $g=p_{1} p_{2} \cdots p_{n} h$, where the elements $p_{1}, p_{2}, \ldots, p_{n}$ belong to either $H_{2}$ or $H_{2}^{\prime}$, and $h \in H_{1}$. So, since $g \in \bar{P}$, we have

$$
h \in \bar{P} \cap H_{1}=\bar{P} \cap \overline{H_{1}} \cap H_{1}=\overline{H_{2}} \cap \overline{H_{1}} \cap H_{1}=\overline{H_{2}} \cap H_{1}=H_{2} \cap H_{1},
$$

because $H_{2}$ and $H_{1}$ are closed. Thus $g \in P$, as required. This proves the claim.

Therefore

$$
\bar{P}=P_{\hat{\mathcal{C}}}=A_{\hat{\mathcal{C}}} \amalg\left(H_{1} \cap H_{2}\right)_{\hat{\mathcal{C}}} \amalg A_{\hat{\mathcal{C}}}^{\prime}=\bar{A} \amalg\left(\overline{H_{1} \cap H_{2}}\right) \amalg \overline{A^{\prime}} .
$$

Also

$$
\overline{H_{2}}=\left(\overline{H_{1} \cap H_{2}}\right) \amalg \bar{A} \text { and } \overline{H_{2}^{\prime}}=\left(\overline{H_{1} \cap H_{2}}\right) \amalg \overline{A^{\prime}} .
$$

We deduce that

$$
\overline{H_{2}} \cap \overline{H_{2}^{\prime}}=\overline{H_{1} \cap H_{2}} .
$$


Thus, since $\overline{H_{1}} \cap \overline{H_{2}}=\overline{H_{1}} \cap \overline{H_{2}^{\prime}}$, we obtain

$$
\overline{H_{1}} \cap \overline{H_{2}}=\overline{H_{1}} \cap \overline{H_{2}} \cap \overline{H_{1}} \cap \overline{H_{2}^{\prime}}=\overline{H_{1} \cap H_{2}} .
$$

This verifies (2.2), as required.

Corollary 2.4. Let $H$ be a finite subgroup of $R$ and let $\Phi$ be a normal free subgroup $\Phi$ of $R$ which is open, i.e., $R / \Phi \in \mathcal{C}$. Then $\overline{C_{\Phi}(H)}=C_{\bar{\Phi}}(H)$.

Proof. Observe that $H \in \mathcal{C}$ since $H$ is isomorphic to a subgroup of $R / \Phi$. We use induction on the order of $H$. Assume first that $H$ has prime order $p$. Then we may assume that $R=H \Phi=\Phi \rtimes H$, because $H \Phi$ is open in $R$, so that its pro- $\mathcal{C}$ topology coincides with the topology induced by the pro- $\mathcal{C}$ topology of $R$, and $(H \Phi)_{\hat{\mathcal{C}}} \leq R_{\hat{\mathcal{C}}}$. Then the result is the content of Lemma $2.2\left(\mathrm{~b}^{\prime}\right)$.

If $H$ is cyclic and its order is not a prime number, choose a maximal subgroup $M$ of $H$. Then, since $C_{\Phi}(H) \leq C_{\Phi}(M)$, one deduces that $C_{\Phi}(H)=C_{\Phi}(M) \cap C_{\Phi}(H)=$ $C_{C_{\Phi}(M)}(H)$. Note that $C_{\Phi}(M)$ is closed and finitely generated, and therefore so is $C_{\Phi}(M) H$. Hence the induced pro- $\mathcal{C}$ topology on $\Phi H$ is its full pro-C topology (this follows from Corollary 3.3 in [11]). Thus we may assume that $\Phi=C_{\Phi}(M)$ and $R=\Phi \rtimes H$, and therefore $M$ is a finite normal subgroup centralizing $\Phi$. Thus factoring out $M$ and identifying $\Phi$ with its image modulo this factorization it suffices to prove the equality $\overline{C_{\Phi}(H / M)}=C_{\bar{\Phi}}(H / M)$. Since the order of $H / M$ smaller than the order of $H$, the result follows from the induction hypothesis.

If $H$ is noncyclic, take $M_{1}$ and $M_{2}$ to be two distinct maximal subgroups of $H$. By Proposition 2.3

$$
\overline{C_{\Phi}(H)}=\overline{C_{\Phi}\left(M_{1}\right) \cap C_{\Phi}\left(M_{2}\right)}=\overline{C_{\Phi}\left(M_{1}\right)} \cap \overline{C_{\Phi}\left(M_{2}\right)} .
$$

Now, by the induction hypothesis, the latter expression coincides with $C_{\bar{\Phi}}\left(M_{1}\right) \cap$ $C_{\bar{\Phi}}\left(M_{2}\right)=C_{\bar{\Phi}}(H)$, as needed.

In [5], Marshall Hall proved that a finitely generated subgroup $H$ of a free abstract group $\Phi$ is closed in the profinite topology of $\Phi$. It easily follows that a finitely generated subgroup of a virtually free abstract group $R$ is automatically closed in the profinite topology of $R$. Therefore we deduce:

Corollary 2.5. (Proposition 2.4 in [18]) Let $R$ be a finitely generated virtually free (or free-by-finite) abstract group. Let $\mathrm{H}_{1}$ and $\mathrm{H}_{2}$ be finitely generated subgroups of $R$. Then

$$
\overline{H_{1} \cap H_{2}}=\overline{H_{1}} \cap \overline{H_{2}},
$$

where if $X \subseteq R$, then $\bar{X}$ denotes the closure of $X$ in the profinite completion $\hat{R}$ of $R$.

Theorem 2.6. Let $R$ be a finitely generated free-by-C abstract group. Consider a finitely generated subgroup $H$ of $R$ which is closed in the pro-C topology of $R$. Then

$$
\overline{N_{R}(H)}=N_{R_{\hat{\mathcal{C}}}}(\bar{H})
$$


Proof. Obviously $\overline{N_{R}(H)} \leq N_{R_{\hat{\mathcal{C}}}}(\bar{H})$. We need to prove the opposite containment.

We continue with the notation of sections 0 and 1. By Lemma 1.5, we have that $R=\Pi_{1}^{\text {abs }}(\mathcal{G}, \Delta)=\Pi^{\text {abs }}$, where $(\mathcal{G}, \Delta)$ is a graph of finite groups in $\mathcal{C}$ over a finite graph $\Delta$. Let $S^{\text {abs }}$ be the standard tree associated with this graph of groups and let $S$ be the standard pro- $\mathcal{C}$ tree associated with $(\mathcal{G}, \Delta)$, considered as a graph of pro- $\mathcal{C}$ groups.

Case 1. $H$ is infinite. By Proposition 1.6, $S^{\text {abs }}$ has a unique minimal $H$-invariant subtree $D^{\text {abs }}$ and its closure $D=\overline{D^{\text {abs }}}$ in $S$ is the unique minimal $\bar{H}$-invariant subtree of $S$.

If $a \in N_{R_{\hat{\mathcal{C}}}}(\bar{H})$, then $a D=D$, because $a D$ is also a minimal $\bar{H}$-invariant pro-C subtree of $S$. In other words, $N_{R_{\hat{\mathcal{C}}}}(\bar{H})$ acts on $D$; in particular $\overline{N_{R_{\hat{\mathcal{C}}}}(H)}$ acts on $D$. Similarly, $N_{R}(H)$ acts on $D^{\text {abs }}$. Next we claim that the natural epimorphism of graphs

$$
N_{R}(H) \backslash D^{\mathrm{abs}} \longrightarrow N_{R_{\hat{\mathcal{C}}}}(\bar{H}) \backslash D
$$

is injective. Let $\tilde{s}=s \Pi^{\mathrm{abs}}(m), \tilde{s}^{\prime}=s^{\prime} \Pi^{\mathrm{abs}}(m) \in D^{\mathrm{abs}}\left(s, s^{\prime} \in R, m \in \Delta\right)$, and assume there exists $a \in N_{R_{\hat{\mathcal{C}}}}(\bar{H})$ such that $\tilde{s}^{\prime}=a \tilde{s}$. Then $s^{\prime-1} a s \Pi^{\mathrm{abs}}(m)=$ $\Pi^{\mathrm{abs}}(m)$, i.e., $s^{\prime-1}$ as $\in \Pi^{\mathrm{abs}}(m) \leq \Pi^{\mathrm{abs}}=R$. Therefore, by Lemma 2.1 ,

$$
a \in R \cap N_{R_{\hat{\mathcal{C}}}}(\bar{H})=N_{R}(H),
$$

proving the claim.

Since $H \backslash D^{\text {abs }}$ is finite, so is $N_{R}(H) \backslash D^{\text {abs }}$. Choose $m \in \Delta$, and $t_{1}=1 \Pi^{\text {abs }}(m)$, $t_{2}, \ldots, t_{r} \in D^{\text {abs }}$ such that

$$
D^{\mathrm{abs}}=N_{R}(H) t_{1} \bullet \cdots \bullet N_{R}(H) t_{r} .
$$

Since this union is finite, taking closures we have

$$
D=\overline{N_{R}(H)} t_{1} \cup \cdots \cup \overline{N_{R}(H)} t_{r}=N_{R_{\hat{\mathcal{C}}}}(\bar{H}) t_{1} \cup \cdots \cup N_{R_{\hat{\mathcal{C}}}}(\bar{H}) t_{r}
$$

(the last equality holds since $\overline{N_{R}(H)} \leq N_{R_{\hat{\mathcal{C}}}}(\bar{H})$ ). By the claim these unions are disjoint. So, in particular, $\overline{N_{R}(H)} t_{1}=N_{R_{\hat{\mathcal{C}}}}(\bar{H}) t_{1}$. Hence, if $a \in N_{R_{\hat{\mathcal{C}}}}(\bar{H})$, there exists $b \in \overline{N_{R}(H)}$ such that $a \Pi^{\mathrm{abs}}(m)=b \Pi^{\mathrm{abs}}(m)$. Therefore $b^{-1} a=x \in$ $\Pi^{\mathrm{abs}}(m) \leq R$. Using Lemma 2.1,

$$
x \in R \cap N_{R_{\hat{\mathcal{C}}}}(\bar{H})=N_{R}(H) .
$$

Thus $a \in \overline{N_{R}(H)}$. This proves that $N_{R_{\hat{\mathcal{C}}}}(\bar{H}) \leq \overline{N_{R}(H)}$, as required.

Case 2. $H$ is finite. Observe that $C_{R_{\hat{\mathcal{C}}}}(H)$ is the kernel of the natural homomorphism $N_{R_{\hat{\mathcal{C}}}}(H) \longrightarrow \operatorname{Aut}(H)$; therefore, since $\operatorname{Aut}(H)$ is finite, $C_{R_{\hat{\mathcal{C}}}}(H)$ has finite index in $N_{R_{\hat{\mathcal{C}}}}(H)$, so that $N_{R_{\hat{\mathcal{C}}}}(H)$ is finite if and only if $C_{R_{\hat{\mathcal{C}}}}(H)$ is finite. Similarly, $N_{R}(H)$ is finite if and only if $C_{R}(H)$ is finite.

Subcase $2(\mathrm{a}) . N_{R}(H)$ is finite. Let $\Phi$ be a normal free abstract subgroup of $R$ such that $R / \Phi \in \mathcal{C}$. Then $N_{\Phi}(H)=\Phi \cap N_{R}(H)=1$; in particular $C_{\Phi}(H)=1$. By Corollary 2.4, $C_{\Phi_{\hat{\mathcal{C}}}}(H)=1$. Hence $N_{R_{\hat{\mathcal{C}}}}(H)$ is finite. Then, by Theorem 3.10 
in [20], $N_{R_{\hat{\mathcal{C}}}}(H)$ is conjugate to a subgroup of some vertex group $\Pi(v)=\Pi^{\text {abs }}(v)$, so that we may assume that it is contained in $\Pi(v)$. Thus

$$
N_{R_{\hat{\mathcal{C}}}}(H)=N_{\Pi(v)}(H)=N_{R}(H)=\overline{N_{R}(H)},
$$

proving the result in this case.

Subcase $2(\mathrm{~b}) . N_{R}(H)$ is infinite. Hence so is $C_{\Phi}(H)$. Since $R$ is finitely generated, so is $\Phi$. Note that $C_{\Phi}(H)$ is the subgroup of elements of the free group $\Phi$ fixed by the finite group $H$ (as a group of automorphisms). Therefore $C_{\Phi}(H)$ is a free factor of $\Phi$ (cf. Theorem 2 in [3]). Hence $C_{\Phi}(H)$ is finitely generated; it is also closed in the pro- $\mathcal{C}$ topology of $\Phi$, and so of $R$ (cf. Corollary 3.1.6 (b) in [14]). It follows that $N_{R}(H)$ is finitely generated and closed in the pro- $\mathcal{C}$ topology of $R$. Therefore we may invoke Proposition 1.6 to see that there is a unique minimal $N_{\Phi}(H)$-invariant subtree $D^{\text {abs }}$ of $S^{\text {abs }}$ whose closure $D=\overline{D^{\text {abs }}}$ is the unique minimal $\overline{N_{\Phi}(H)}$-invariant subtree of $S$. Furthermore, $N_{\Phi}(H) / D^{\text {abs }}=\overline{N_{\Phi}(H)} / D$ is finite.

We claim that $N_{R_{\hat{\mathcal{C}}}}(\bar{H})$ also acts on $D$ : since $\overline{N_{\Phi}(H)}$ is infinite and the stabilizers of vertices of $D$ are finite, we can apply Lemma 1.2 to conclude that $D$ is the unique $\overline{C_{\Phi}(H)}$-invariant pro- $\mathcal{C}$ subtree of $S$. Now, by Corollary $2.4 C_{\Phi_{\hat{\mathcal{C}}}}(H)=$ $\overline{C_{\Phi}(H)}$, and again by Lemma $1.2, D$ is in fact the unique minimal $N_{R_{\hat{\mathcal{C}}}}(H)$-invariant subtree of $S$. This proves the claim.

Next we proceed as in Case 1. One sees, as in that case, that the natural map $N_{R}(H) \backslash D^{\text {abs }} \longrightarrow N_{R_{\hat{\mathcal{C}}}}(H) \backslash D$ is injective; one deduces that $\overline{N_{R}(H)} t_{1}=N_{R_{\hat{\mathcal{C}}}}(H) t_{1}$ where $t_{1}=1 \Pi^{\text {abs }}(m)$, for some $m \in \Delta$; and this implies that $N_{R_{\hat{\mathcal{C}}}}(H) \leq \overline{N_{R}(H)}$, as needed.

Lemma 2.7. Let $R$ be a finitely generated abstract free-by-C group. Say $\Phi$ is a normal subgroup of $R$ which is free and such that $R / \Phi \in \mathcal{C}$. Let $H$ be a cyclic subgroup of $\Phi$. Then

$$
C_{R}(H)=C_{R}(C l(H)),
$$

where $C l(H)$ denotes the closure of $H$ in the pro-C topology of $R$.

Proof. Note that $\mathrm{Cl}(H)$ is also the closure of $H$ in the pro- $\mathcal{C}$ topology of $\Phi$, since $\Phi$ is closed in $R$. By Proposition 3.4 in [11], $C l(H)$ is cyclic and contains $H$ as a subgroup of finite index. Say $C l(H)=\langle x\rangle$ and $H=\left\langle x^{n}\right\rangle$. Now, if $a \in R$ and $a^{-1} x^{n} a=x^{n}$, then both $a^{-1} x a$ and $x$ are $n$-th roots of $x^{n}$. Since in a free abstract group $n$-th roots are unique, we deduce that $a^{-1} x a=x$. The result follows.

Corollary 2.8. Let $R$ be a finitely generated abstract group that contains a normal subgroup $\Phi$ which is free and such that $R / \Phi \in \mathcal{C}$. If $H$ is an infinite cyclic closed subgroup of $R$, then

$$
C_{R_{\hat{\mathcal{C}}}}(\bar{H})=\overline{C_{R}(H)}
$$

Moreover, this equality also holds for any (not necessarily closed) cyclic subgroup $H$ of $\Phi$. 
Proof. Consider the natural homomorphism

$$
\varphi: N_{R}(H) \longrightarrow \operatorname{Aut}(H) \cong \mathbf{Z} / 2 \mathbf{Z} .
$$

Then $\operatorname{Ker}(\varphi)=C_{R}(H)$. Note that

$$
\overline{C_{R}(H)} \leq C_{R_{\hat{\mathcal{C}}}}(\bar{H}) \leq N_{R_{\hat{\mathcal{C}}}}(\bar{H})=\overline{N_{R}(H)}
$$

(for the last equality we use Theorem 2.6). Since the index of $\overline{C_{R}(H)}$ in $\overline{N_{R}(H)}$ is at most 2, the result follows immediately: suppose $C_{R_{\hat{\mathcal{C}}}}(\bar{H})=\overline{N_{R}(H)}$ and let $r \in N_{R}(H)$; then $r \in C_{R_{\hat{\mathcal{C}}}}(\bar{H})$, and so $r \in C_{R}(H)$, i.e., $C_{R}(H)=N_{R}(H)$. Hence $\overline{C_{R}(H)}=C_{R_{\hat{C}}}(\bar{H})$.

Assume now that $H$ is a cyclic subgroup of $\Phi$, not necessarily closed. By Lemma 2.7, $C_{R}(H)=C_{R}(C l(H))$. Therefore using the result above for the closed subgroup $C l(H)$,

$$
\overline{C_{R}(H)}=\overline{C_{R}(C l(H))}=C_{R_{\hat{\mathcal{C}}}}(\overline{C l(H)})=C_{R_{\hat{\mathcal{C}}}}(\bar{H}),
$$

since $\bar{H}=\overline{C l(H)}$.

Using the result of $\mathrm{M}$. Hall mentioned above, one deduces immediately the following consequence to Theorem 2.6.

Corollary 2.9. Let $R$ be a finitely generated virtually free (or free-by-finite) abstract group, and let $H$ be a finitely generated subgroup. Then

$$
\overline{N_{R}(H)}=N_{\hat{R}}(\bar{H}) .
$$

\section{Conjugacy $\mathcal{C}$-separability}

An abstract group $R$ is said to be conjugacy $\mathcal{C}$-separable if for any pair of nonconjugate elements $x$ and $y$ of $R$, there is a quotient group of $R$ which is in $\mathcal{C}$, and where the images of $x$ and $y$ are not conjugate. In this section we prove the conjugacy $\mathcal{C}$-separability of a finitely generated free-by- $\mathcal{C}$ abstract group $R$. This generalizes Theorem 1.7 in [17], where it is proved for the class $\mathcal{C}$ of all finite $p$-groups, for a fixed prime $p$. This result was proved by G. Baumslag and T. Taylor when $R$ is a free abstract group (cf. Proposition 4.8 in [8]). Our result assumes the theorem of Baumslag-Taylor and it is based on Theorem 2.6 above.

Note that if $R$ is residually $\mathcal{C}$, then conjugacy $\mathcal{C}$-separability of $R$ means that for any pair of elements of $R$, they are conjugate in $R$ if and only if they are conjugate in the pro- $\mathcal{C}$ completion $R_{\hat{\mathcal{C}}}$ of $R$.

Lemma 3.1. Let $R$ be a finitely generated free-by-C abstract group and let $H$ be a finitely generated closed (in the pro-C topology of $R$ ) subgroup of $R$. Then the pro-C topology of $H$ coincides with the topology induced by the pro-C topology of $R$, i.e., $H_{\hat{\mathcal{C}}}=\bar{H}$. 
Proof. Let $\Phi$ be an abstract free normal subgroup of $R$ such that $R / \Phi \in \mathcal{C}$. Since $\Phi$ is open in $R$ and $\Phi \cap H$ is open in $H$, it suffices to prove that the pro- $\mathcal{C}$ topology of $\Phi$ induces on $\Phi \cap H$ its pro- $\mathcal{C}$ topology (cf. Lemma 3.1.4 (b) in [14]). This is the content of Corollary 3.3 (ii) in [11].

Theorem 3.2. Let $R$ be a finitely generated free-by-C abstract group. Then $R$ is conjugacy $\mathcal{C}$-separable.

Proof. To fix the notation, say that $\Phi \triangleleft R$, where $\Phi$ is an abstract free group such that $R / \Phi \in \mathcal{C}$. By Lemma $0.4, R$ is residually $\mathcal{C}$. Let $x, y \in R$ and let $x^{\gamma}=y$, where $\gamma \in R_{\hat{C}}$. We have to show that $x$ and $y$ are conjugate in $R$. We may assume that $x \neq 1$. Since $R_{\hat{C}}=R \Phi_{\hat{\mathcal{C}}}$, we have $\gamma=r \eta$, for some $\eta \in \Phi_{\hat{\mathcal{C}}}$ $r \in R$. So replacing $x$ by $x^{r}$ and $\gamma$ by $\eta$, we may assume that $\gamma$ is in $\Phi_{\hat{\mathcal{C}}}$. Then $y \in\langle x\rangle \Phi_{\hat{\mathcal{C}}} \cap R=\langle x\rangle \bar{\Phi} \cap R=\langle x\rangle(\bar{\Phi} \cap R)=\langle x\rangle \Phi$. Hence, from now on, we may also assume that $R=\langle x\rangle \Phi$. Note that $R_{\hat{\mathcal{C}}}=\langle x\rangle \Phi_{\hat{\mathcal{C}}}$. Since $R_{\hat{\mathcal{C}}} / \Phi_{\hat{\mathcal{C}}}$ is abelian, we have $x^{-1} \gamma^{-1} x \gamma \in \Phi_{\hat{\mathcal{C}}}$, i.e., $x \Phi_{\hat{\mathcal{C}}}=x^{\gamma} \Phi_{\hat{\mathcal{C}}}$. On the other hand, the natural map $\rho: R / \Phi \longrightarrow R_{\hat{\mathcal{C}}} / \Phi_{\hat{\mathcal{C}}}$ is a bijection. Since $\rho(y \Phi)=y \Phi_{\hat{\mathcal{C}}}=x^{\gamma} \Phi_{\hat{\mathcal{C}}}=x \Phi_{\hat{\mathcal{C}}}=\rho(x \Phi)$, we deduce that $y \Phi=x \Phi$. From now on we assume that

$$
R=\langle x\rangle \Phi, \quad y=x^{\gamma} \in R, \text { with } \gamma \in \Phi_{\hat{\mathcal{C}}}, \text { and } y \Phi=x \Phi .
$$

Now we distinguish two cases.

Case 1. The order of $x$ is infinite. Let $n$ be a positive integer such that $x^{n} \in \Phi$. So $y^{n} \in \Phi$ and $y^{n}=\left(x^{n}\right)^{\gamma}$. From the Baumslag-Taylor result mentioned above we deduce that $y^{n}$ and $x^{n}$ are conjugate in $\Phi$. Say $f^{-1} x^{n} f=y^{n}$, where $f \in \Phi$. Replacing $x$ with $f x f^{-1}$, we may assume that $y^{n}=x^{n}$. Therefore $\gamma \in C_{R_{\hat{\mathcal{C}}}}\left(x^{n}\right)$. Since $x^{n} \in \Phi$, we may apply Corollary 2.8 to get that $C_{R_{\hat{\mathcal{C}}}}\left(x^{n}\right)=\overline{C_{R}\left(x^{n}\right)}$. Thus we have $x, y, \gamma \in \overline{C_{R}\left(x^{n}\right)}$.

Note that $C_{R}\left(x^{n}\right) \cap \Phi=C_{\Phi}\left(x^{n}\right)$. Since $x^{n} \neq 1$ and $\Phi$ is free, $C_{\Phi}\left(x^{n}\right)$ is cyclic, say $C_{\Phi}\left(x^{n}\right)=\langle z\rangle$ and $z^{m}=x^{n}$, for some natural number $m$. Using the uniqueness of $m$ th roots in $\Phi$, we get that $C_{R}\left(x^{n}\right)=C_{R}(z)$ (see the argument in the proof of Lemma 2.7). Hence $x \in C_{R}(z)$, i.e., $x$ and $z$ commute.

Since $R=\langle x\rangle \Phi$, we obtain that $C_{R}\left(x^{n}\right)=\langle x\rangle C_{\Phi}\left(x^{n}\right)=\langle x\rangle\langle z\rangle$. Therefore $C_{R}\left(x^{n}\right)$ is abelian, and hence so is $\overline{C_{R}\left(x^{n}\right)}$. This implies that $x=y$. Thus the result follows in this case.

Case 2. The order of $x$ is finite. Observe that $\langle x\rangle$ is isomorphic to a subgroup of $R / \Phi$, and so $\langle x\rangle \in \mathcal{C}$. We proceed by induction on the order of $x$.

Subcase 2 (a). The order of $x$ is $p$, a prime. As in the proof of Lemma 2.2, we have

$$
R=\langle x\rangle \Phi=\left[*_{i=1}^{n}\left(C_{i} \times \Phi_{i}\right)\right] * L, \quad \text { and } \quad R_{\hat{\mathcal{C}}}=\left[\coprod_{i=1}^{n}\left(C_{i} \times\left(\Phi_{i}\right)_{\hat{\mathcal{C}}}\right)\right] \amalg L_{\hat{\mathcal{C}}},
$$

where $L$ and each $\Phi_{i}$ are free groups and the $C_{i}$ are groups of order $p$. Since every finite subgroup of $R$ of order $p$ is conjugate in $R$ to one of the $C_{i}$ (cf. [16], Part I, 
Corollary 1 of Proposition 2), we can assume that $C_{1}=\langle y\rangle$. Since $R=\langle x\rangle \Phi$, there exists some $f \in \Phi$ such that $\langle x\rangle^{f}=C_{j}$, for some $j$ with $1 \leq j \leq n$. Replacing $x$ with $x^{f}$ and $\gamma$ with $f^{-1} \gamma$, we can assume that $\langle x\rangle=C_{j}$; note that the conditions (3.1) still hold. Since $x$ and $y$ are conjugate in $R_{\hat{\mathcal{C}}}$, we deduce that $x=y$ (cf. Theorem 9.1.12 in [14]). Hence the result holds in this case.

Subcase 2 (b). The order of $x$ is finite but not a prime. Choose a natural number $n$ such that the order of $x^{n}$ is a prime. By the subcase above, replacing $x$ by a certain conjugate in $R$, we may assume that $x^{n}=y^{n}$, and so $\gamma$ centralizes $x^{n}$. Hence $\gamma \in C_{\Phi_{\hat{\mathcal{c}}}}\left(x^{n}\right)=\overline{C_{\Phi}\left(x^{n}\right)}$ (the last equality is the content of Lemma $2.2\left(\mathrm{~b}^{\prime}\right)$ ). Put $H=\langle x\rangle C_{\Phi}\left(x^{n}\right)$. Since $x$ normalizes $C_{\Phi}\left(x^{n}\right), H$ is a subgroup of $R$. By Lemma 2.2, $C_{\Phi}\left(x^{n}\right)$ is a free factor of $\Phi$, and so it is closed in $\Phi$. Hence $C_{\Phi}\left(x^{n}\right)$ is closed in $R$. Since $\langle x\rangle$ is finite, $H$ is closed in $R$, so by Lemma $3.1, H_{\hat{\mathcal{C}}}=\bar{H}$. Therefore, $H_{\hat{\mathcal{C}}}=\bar{H}=\langle x\rangle \overline{C_{\Phi}\left(x^{n}\right)}$. It follows that $x, y \in H$ and $\gamma \in H_{\hat{\mathcal{C}}}$. Hence we may assume that $R=H=\langle x\rangle C_{\Phi}\left(x^{n}\right)$. Moreover, conditions (3.1) still hold, where now $C_{\Phi}\left(x^{n}\right)$ plays the role of $\Phi$. Note that then $\left\langle x^{n}\right\rangle$ is a central subgroup of $R$, and $R /\left\langle x^{n}\right\rangle=\left(\langle x\rangle /\left\langle x^{n}\right\rangle\right) C_{\Phi}\left(x^{n}\right)$, where, with a certain abuse of notation, we identify $C_{\Phi}\left(x^{n}\right)$ with its isomorphic image in $R /\left\langle x^{n}\right\rangle$. Denote by $\tilde{x}$ and $\tilde{y}$ the images of $x$ and $y$ in $R /\left\langle x^{n}\right\rangle$, respectively. So $R /\left\langle x^{n}\right\rangle=\langle\tilde{x}\rangle C_{\Phi}\left(x^{n}\right)$. Note that the order of $\tilde{x}$ is strictly smaller than the order of $x$, namely, $\tilde{y}=\tilde{x}^{\gamma}$, with $\gamma \in C_{\Phi}\left(x^{n}\right)$, and $C_{\Phi}\left(x^{n}\right)$ is a finitely generated free normal subgroup of $R /\left\langle x^{n}\right\rangle$ such that $\left(R /\left\langle x^{n}\right\rangle\right) / C_{\Phi}\left(x^{n}\right) \cong\langle\tilde{x}\rangle \in \mathcal{C}$. By the induction hypothesis, there exists some $f \in C_{\Phi}\left(x^{n}\right)$ such that $\tilde{y}=\tilde{x}^{f}$. Replacing $x$ with $x^{f}$ and $\gamma$ with $f^{-1} \gamma$, we may assume that $\tilde{y}=\tilde{x}$; observe that conditions (3.1) still hold, with $C_{\Phi}\left(\left\langle x^{n}\right\rangle\right)$ playing the role of $\Phi$. Therefore $y=x c$, for some $c \in\left\langle x^{n}\right\rangle$. Since $x C_{\Phi}\left(\left\langle x^{n}\right\rangle\right)=y C_{\Phi}\left(\left\langle x^{n}\right\rangle\right)$, and $C_{\Phi}\left(x^{n}\right)$ is a free group, we have $c=1$. Thus $x=y$, and the result follows.

\section{References}

[1] Dicks, W. And Dunwoody, M. J.: Groups acting on graphs. Cambridge Studies in Advanced Mathematics 17, Cambridge University Press, Cambridge, 1989.

[2] Dyer, J. L.: Separating conjugates in free-by-finite groups. J. London Mat. Soc. (2) 20 (1979), 215-221.

[3] Dyer, J. L. And Scott, P.: Periodic automorphisms of free groups. Comm. Alg. 3 (1975), 195-201.

[4] Gildenhuys, D. And Ribes, L.: Profinite groups and Boolean graphs. J. Pure Appl. Algebra 12 (1978), 21-47.

[5] Hall, M., JR.: A topology for free groups and related groups. Ann. Math. (2) 52 (1950), 127-139.

[6] Herfort, W. and Ribes, L.: Torsion elements and centralizers in free products of profinite groups. J. Reine Angew. Math. 358 (1985), 155-161.

[7] Karrass, A., Pietrowski, A. And Solitar, D.: Finite and infinite cyclic extensions of free groups. J. Austrl. Math. Soc. 16 (1973), 458-466.

[8] Lyndon, R. C. And Schupp, P. E.: Combinatorial group theory. Ergebnisse der Mathematik und ihrer Grenzgebiete 89, Springer-Verlag, Berlin-New York, 1977. 
[9] Magnus, W., Karrass, A. And Solitar, D.: Combinatorial group theory: Presentations of groups in terms of generators and relations. J. Wiley and Sons, New York-London-Sydney, 1966.

[10] Ribes, L., Segal, D. And ZalesskiǏ, P.: Conjugacy separability and free products of groups with cyclic amalgamation. J. London Math. Soc. (2) 57 (1998), 609-628.

[11] Ribes, L. And ZalesskiĬ, P.: The pro- $p$ topology of a free group and algorithmic problems in semigroups. Internat. J. Algebra Comput. 4 (1994), 359-374.

[12] Ribes, L. And Zalesskiŭ, P.: Conjugacy separability and amalgamated free products of groups. J. Algebra 179 (1996), 751-774.

[13] Ribes, L. And Zalesskil, P.: Profinite topologies in free products of groups. Internat. J. Algebra Comput. 14 (2004), 751-772.

[14] Ribes, L. and Zalesskil, P.: Profinite groups. Second edition. Results in Mathematics and Related Areas, 3rd Series, A Series of Modern Surveys in Mathematics 40, Springer-Verlag, Berlin, 2010.

[15] Serre, J.-P.: Cohomologie galoisienne. Fith edition. Lecture Notes in Mathematics 5, Springer-Verlag, Berlin, 1994.

[16] Serre, J.-P.: Trees. Springer-Verlag, Berlin-New York, 1980.

[17] Toinet, E.: Conjugacy p-separability of right-angled Artin groups and applications. Groups Geom. Dyn. 7 (2013), no. 3, 751-790.

[18] Wilson, J.S. And ZalesskiĬ, P. A.: Conjugacy separability of certain Bianchi groups and HNN extensions. Math. Proc. Cambridge Philos. Soc. 123 (1998), no. 2, $227-242$.

[19] Zalesskil, P. A.: Profinite groups, without free nonabelian pro- $p$-subgroups, that act on trees. Mat. Sb. 181 (1990), no. 1, 57-67; translation in Math USSR-Sb. 69 (1991), no. 1, 57-67.

[20] Zalesskiľ, P. A. And Mel'nikov, O. V.: Subgroups of profinite groups acting on trees. Mat. Sb. (N.S.) 135 (1988), 419-439; translation in Math USSR Sb. 63 (1989), 405-424.

Received April 9, 2012.

Luis RiBes: School of Mathematics and Statistics, Carleton University, Ottawa, ON, K1S 5B6, Canada.

E-mail: lribes@math.carleton.ca

Pavel A. ZalesskiǏ: Departamento de Matemática, Universidade de Brasília, 70910900, Brasília-DF, Brazil.

E-mail: pz@mat.unb.br

The first author gratefully acknowledges the support of an NSERC research grant, and the second author the support of CNPq. 